\title{
NOvA Reconstruction using Deep Learning
}

Micah Groh

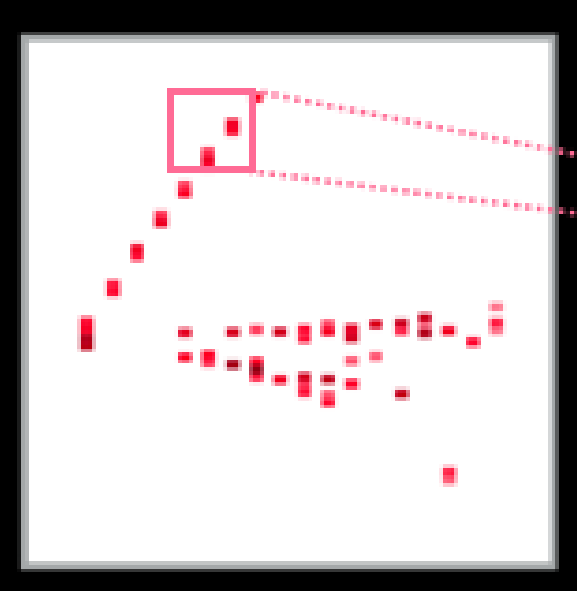

\section{CONVOLUTION}

LAYER

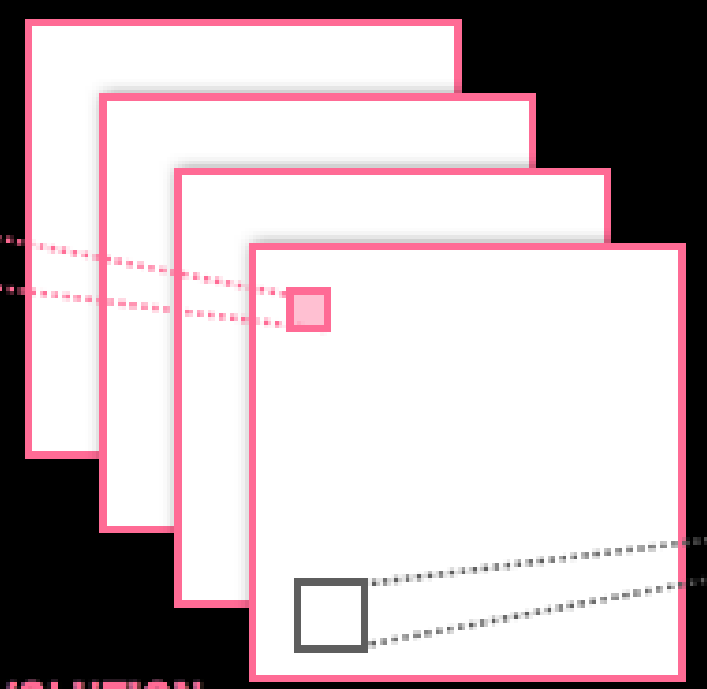

\section{POOLING}

LAYER
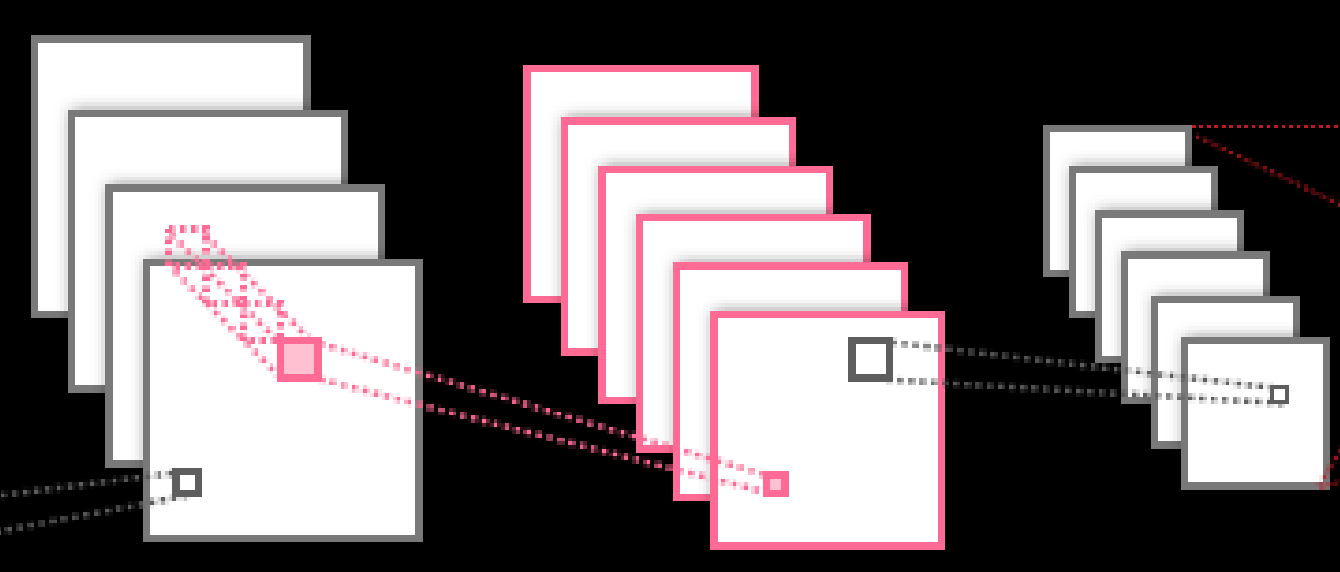

CONVOLUTION

LAYER

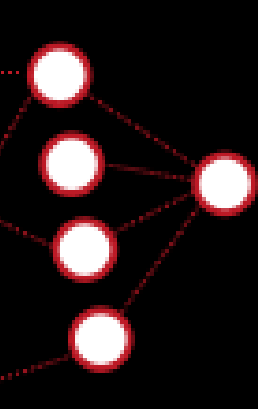

FULLY

CONNECTED LAYER

This document was prepared by [NOvA Collaboration] using the resources of the Fermi National Accelerator Laboratory (Fermilab), a U.S. Department of Energy, Office of Science, HEP User Facility. Fermilab is managed by Fermi Research Alliance, LLC (FRA), acting under Contract No. DE-AC02-07CH11359. 


\section{Convolutional Neural Nets}

Method: Use convolutional neural networks (CNN) to extract features from then classify a "pixel map" made using detector hits from an event.

Why use CNNs?:

1. Designed to optimally use topological features

2. Learn which features have the best discrimination power

3. Decouples the task of classification from the reconstruction inefficiencies

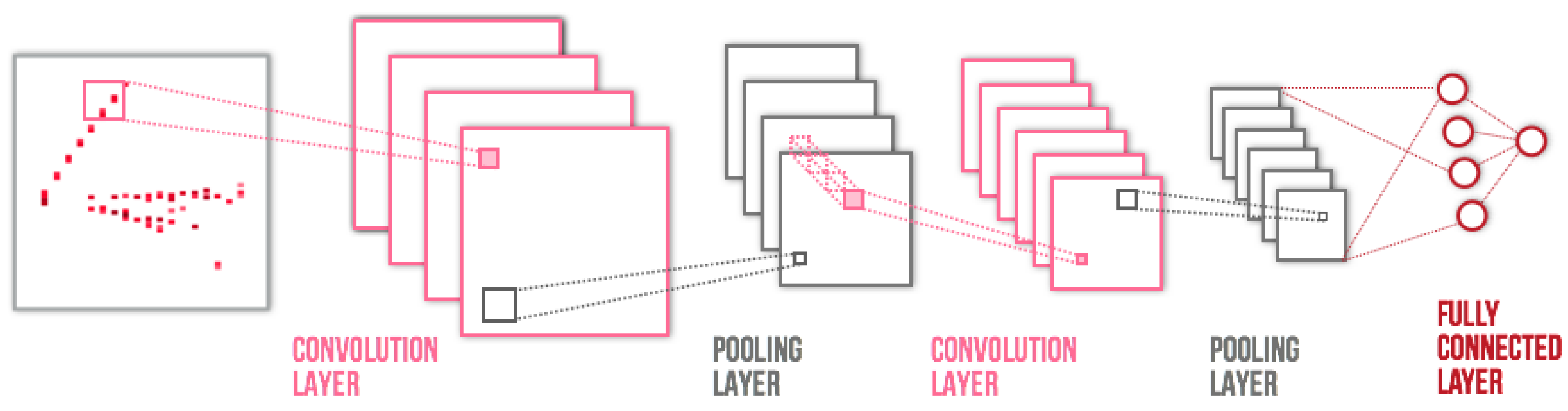




\section{Reconstruction Goals}
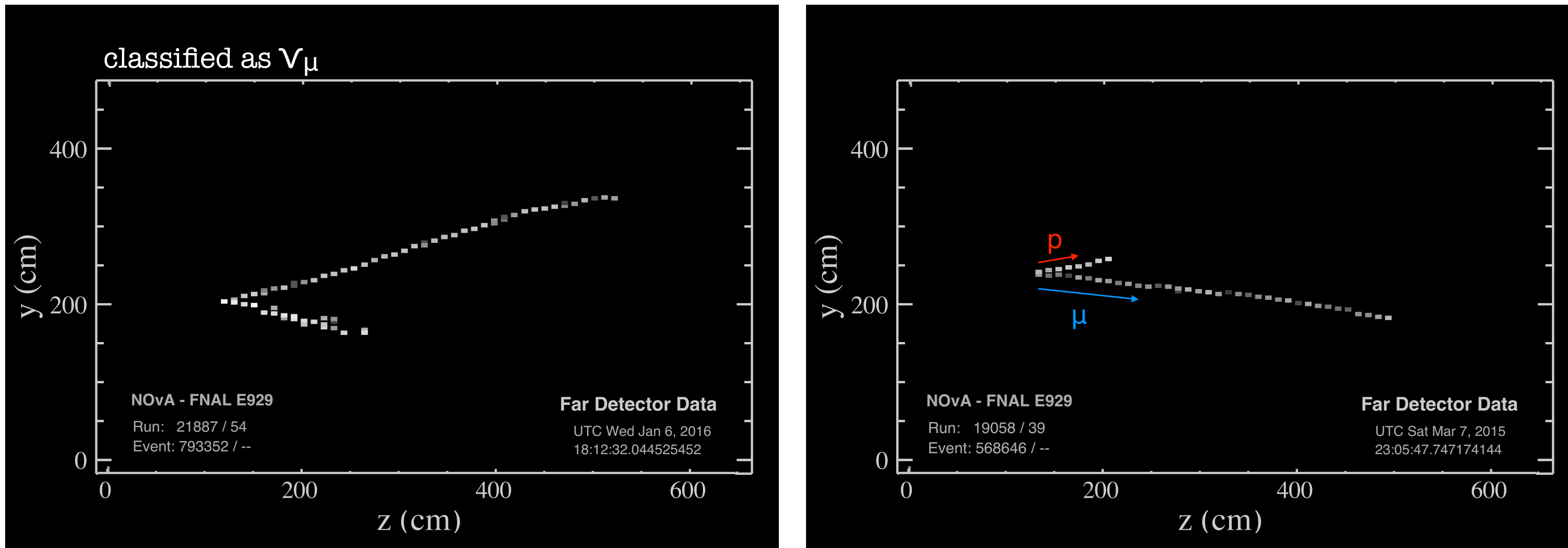

Identify the flavor of a neutrino interaction

Classify particles from a neutrino interaction

\section{Applications:}

Numu Disappearance Nue Appearance NC Disappearance

\section{Applications:}

Nue Energy Estimator PiO Mass Peak Cross Section Measurements 


\section{Event Classification}
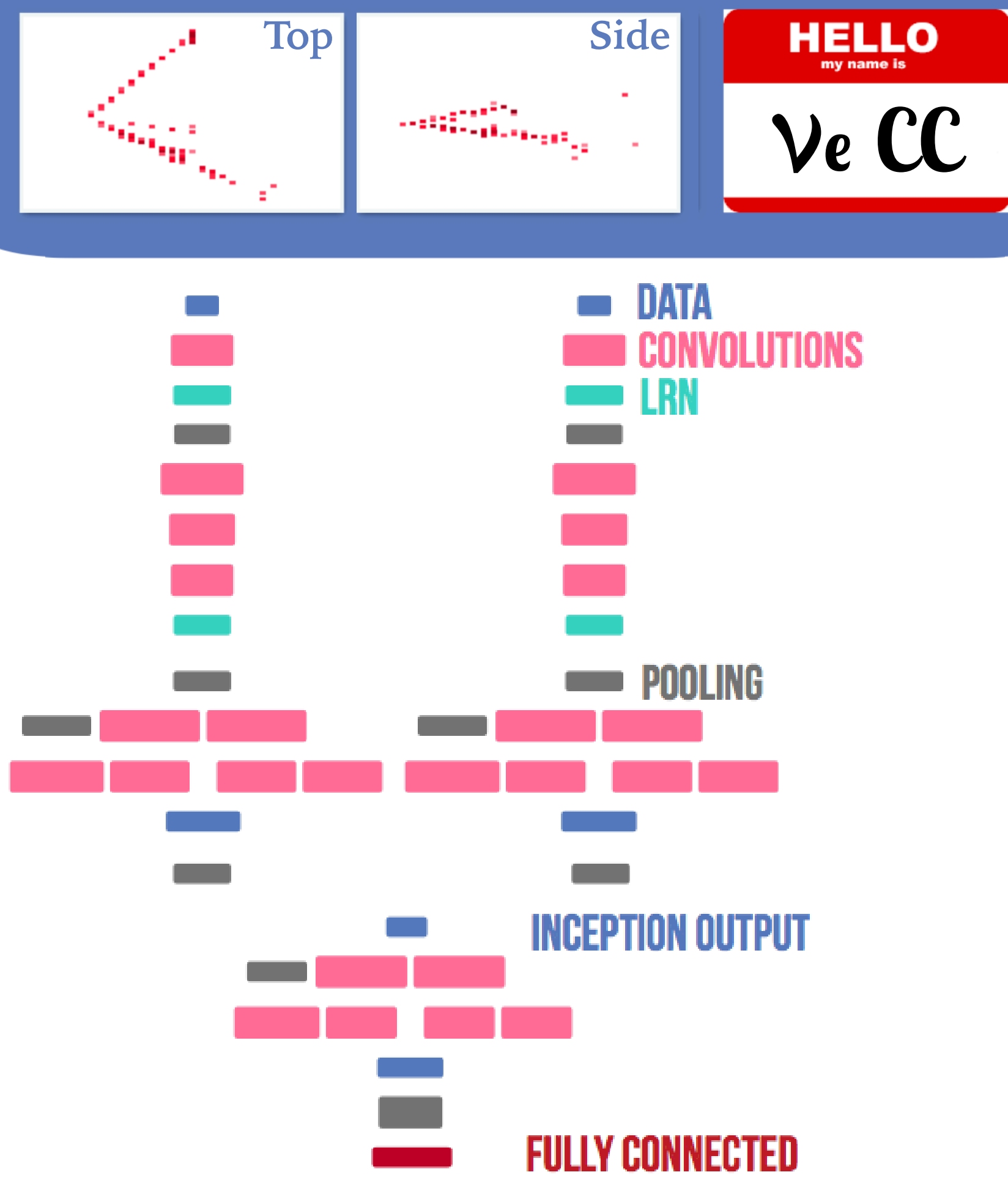

F. Psihas, Ph.D. thesis, Indiana University, 2018, doi:10.2172/1437288.
Classify neutrino events using two tower network, Convolutional Visual Network.

Each view of the event is examined separately for most of feature extraction.

New this analysis:

Updated simulation.

Classification is done using final states.

Network optimizations.

Separate neutrino and antineutrino training. 


\section{Event Classification}
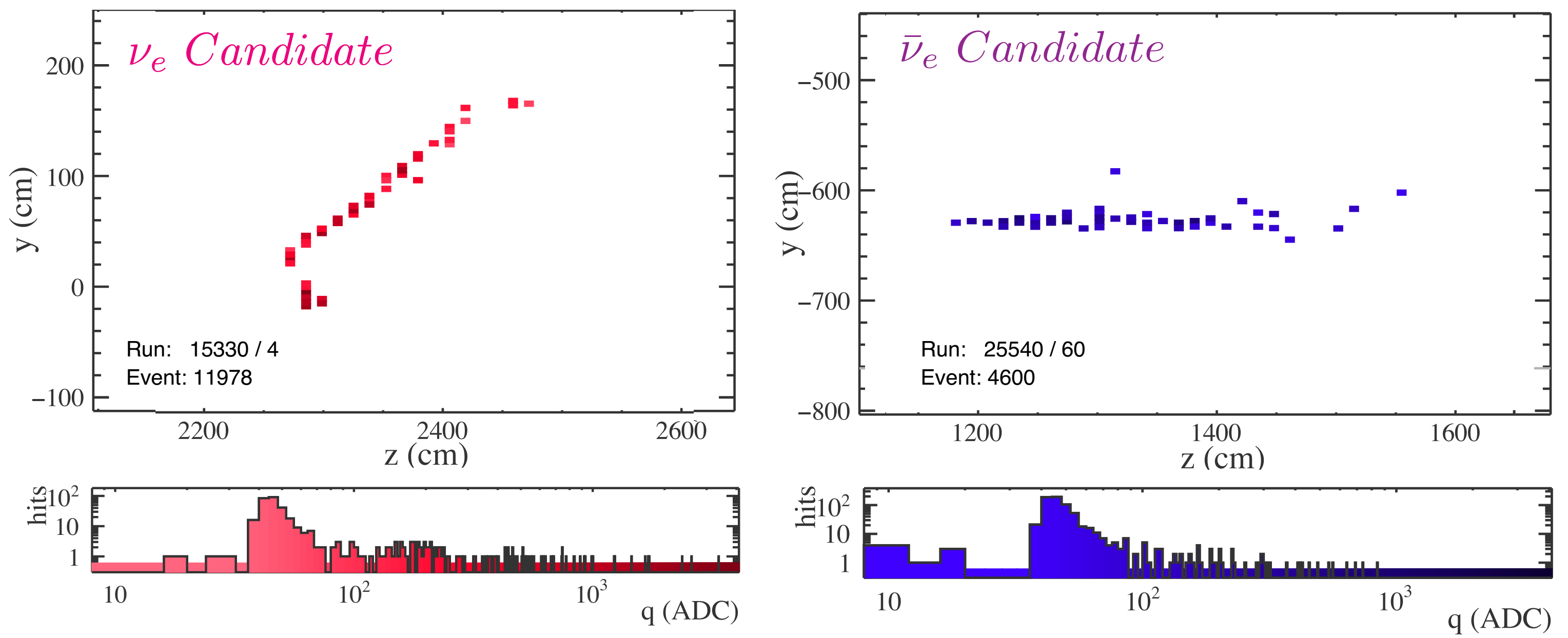

Train on neutrino beam and anti-neutrino beams separately.

Utilize differences in event topology.

\begin{tabular}{|ccc|}
\hline \multicolumn{3}{|c|}{$\bar{\nu}$ Efficiency Improvement } \\
Training Sample (ID > $>.9)$ \\
\hline $\bar{\nu}_{e}$ CC Signal & $\bar{\nu}_{\mu}$ CC Signal & $\bar{\nu}$ NC Signal \\
$14 \%$ & $6 \%$ & $10 \%$ \\
\hline
\end{tabular}




\section{Event Classification}

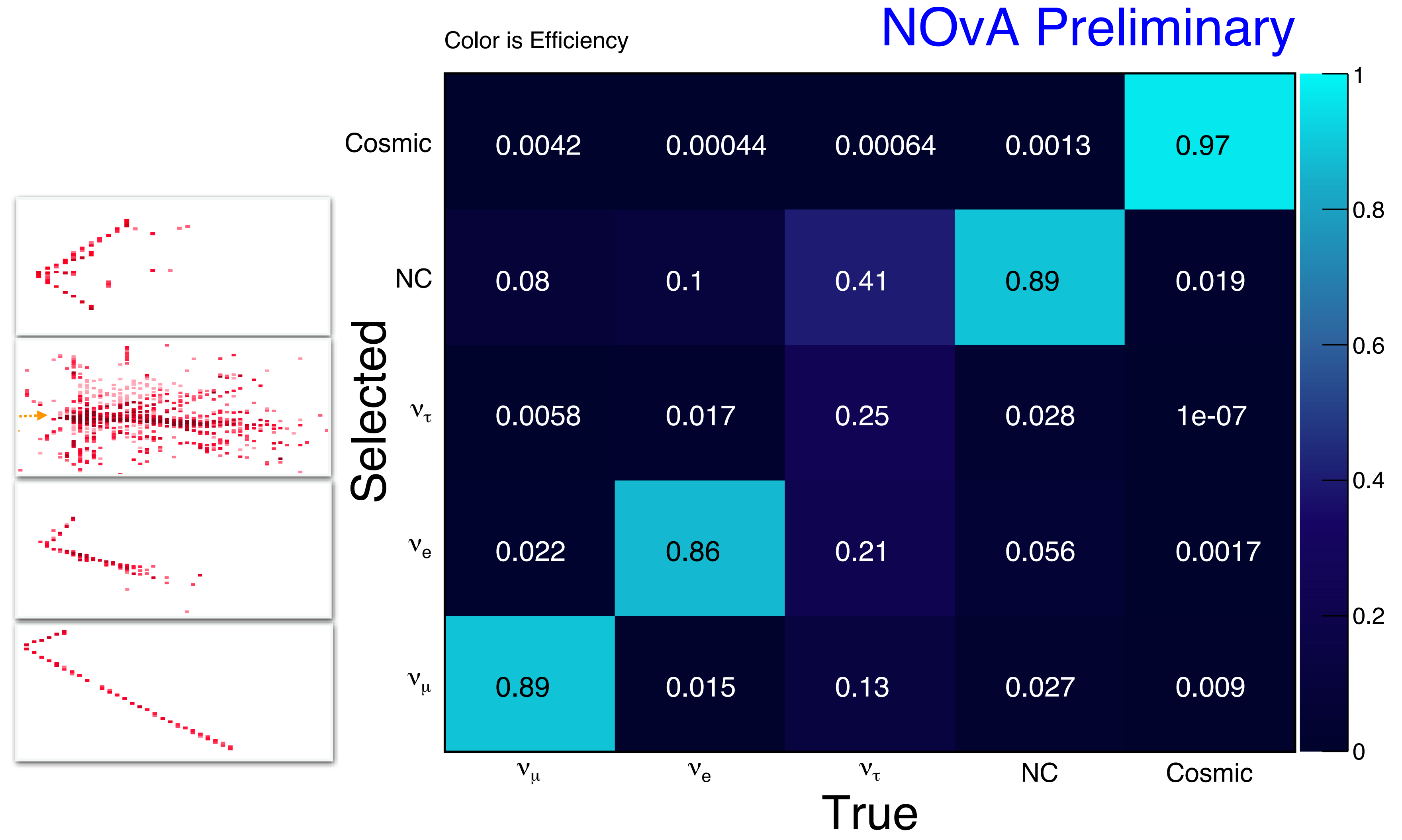




\section{Example Data Check: MRE}
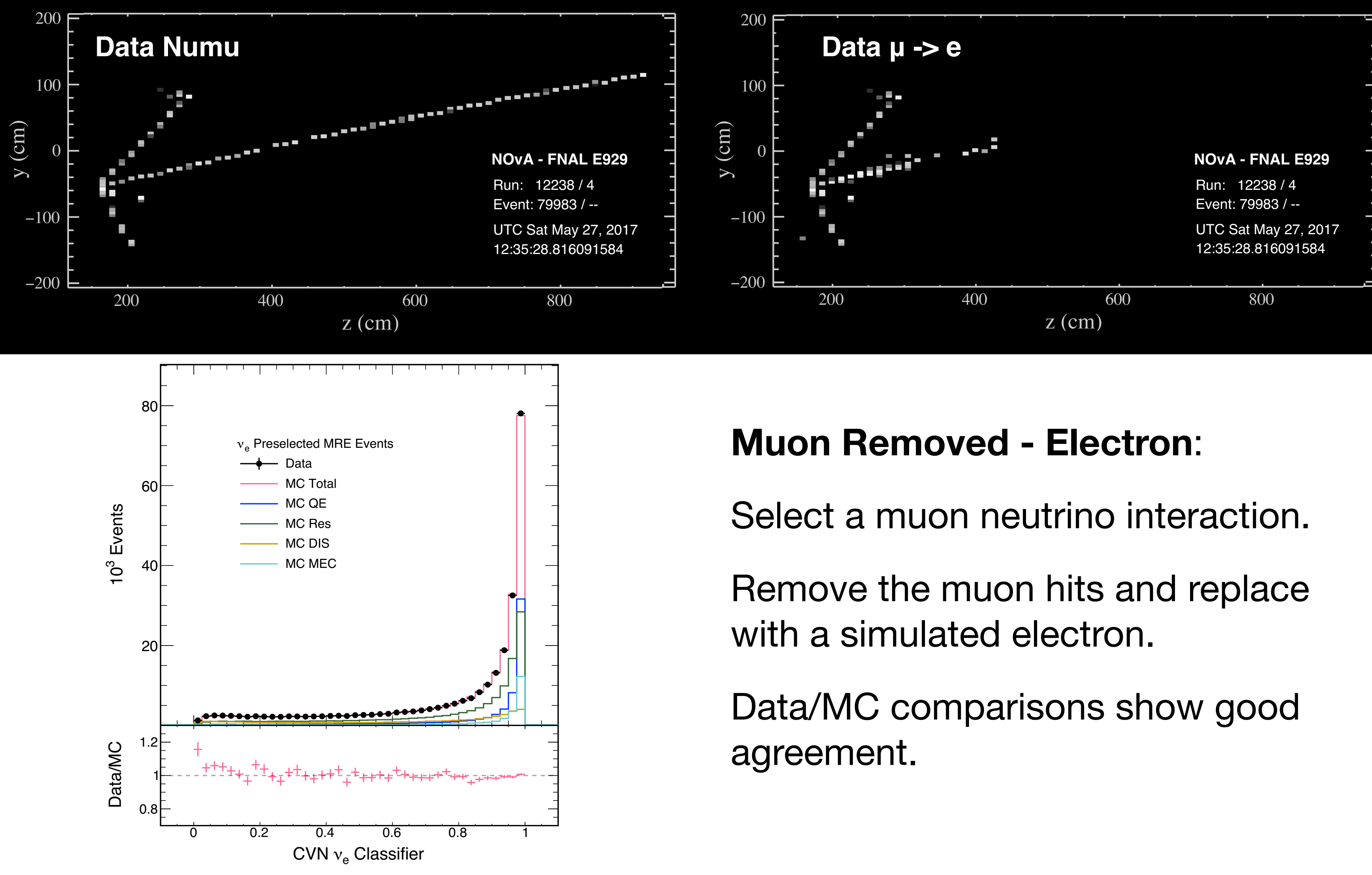

\section{Muon Removed - Electron:}

Select a muon neutrino interaction.

Remove the muon hits and replace with a simulated electron.

Data/MC comparisons show good agreement. 


\section{Particle Classification}
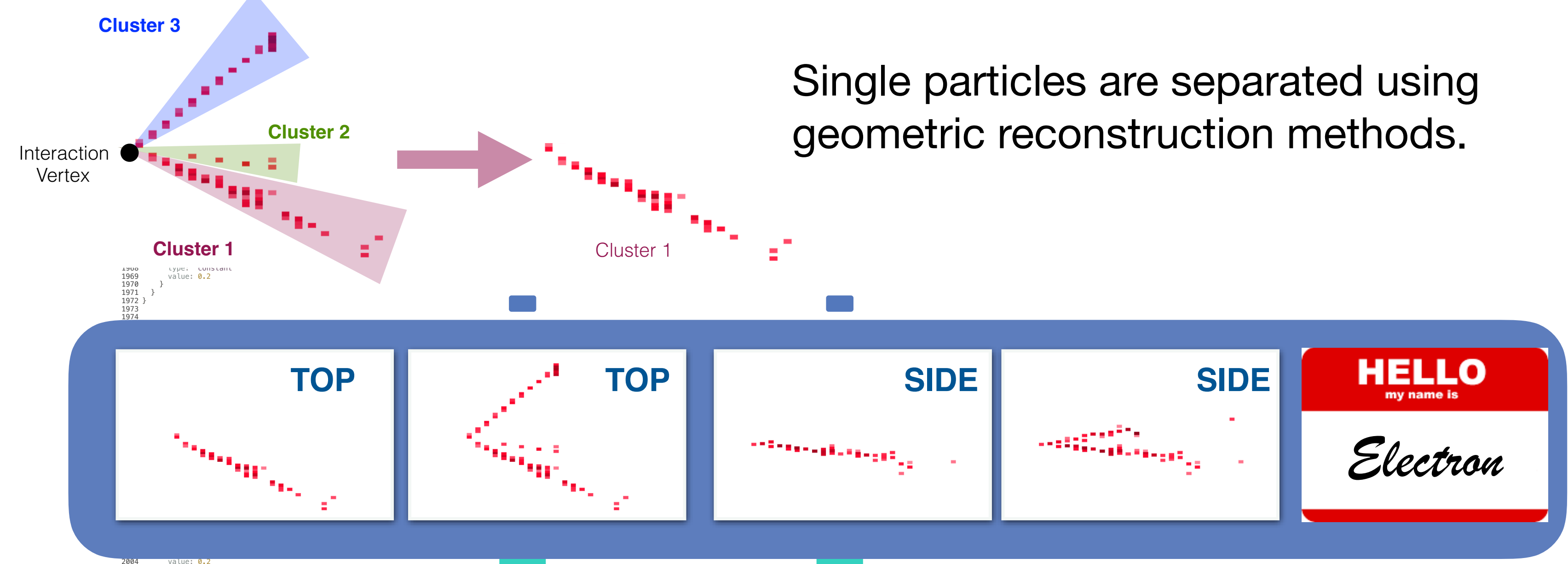

Classify particles using both views of the particle and both views of the entire event.

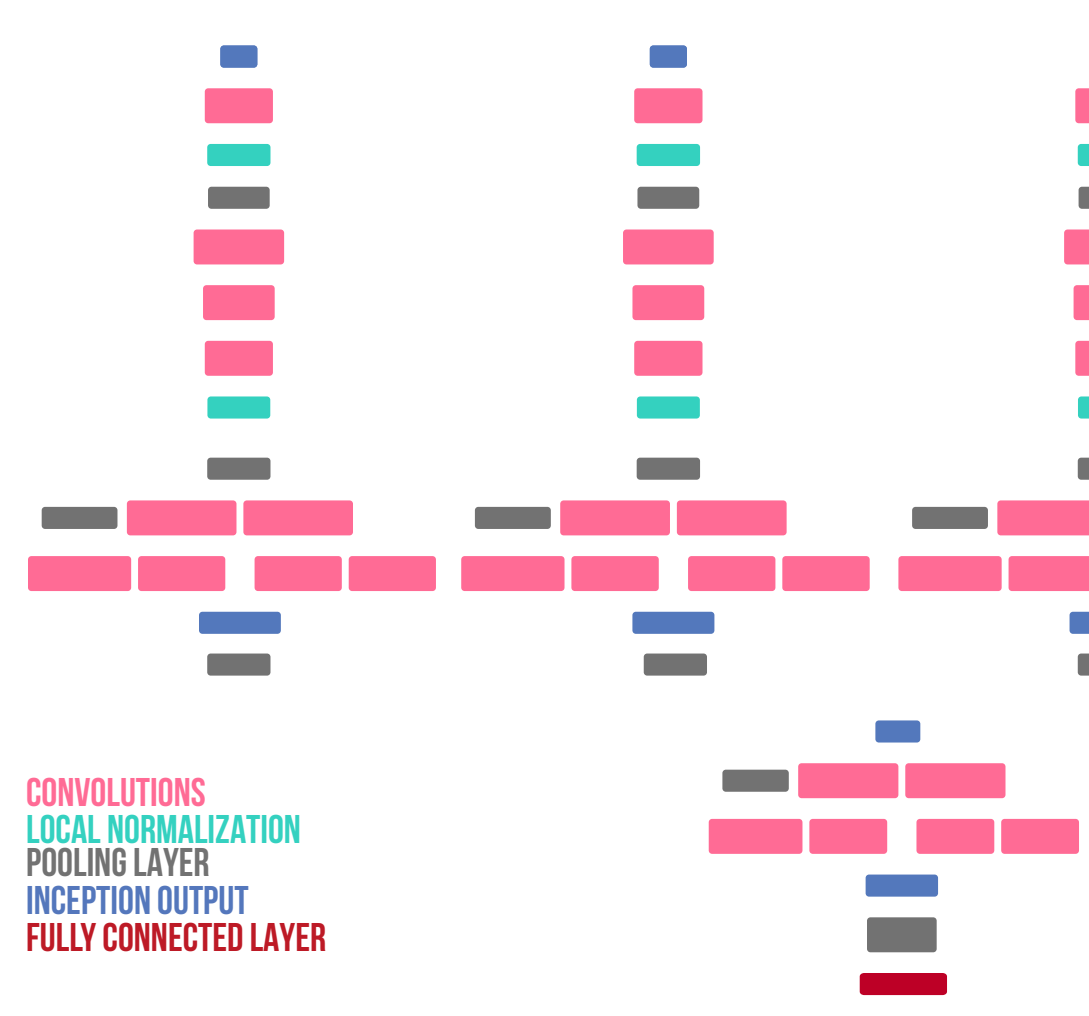




\section{Particle Classification}

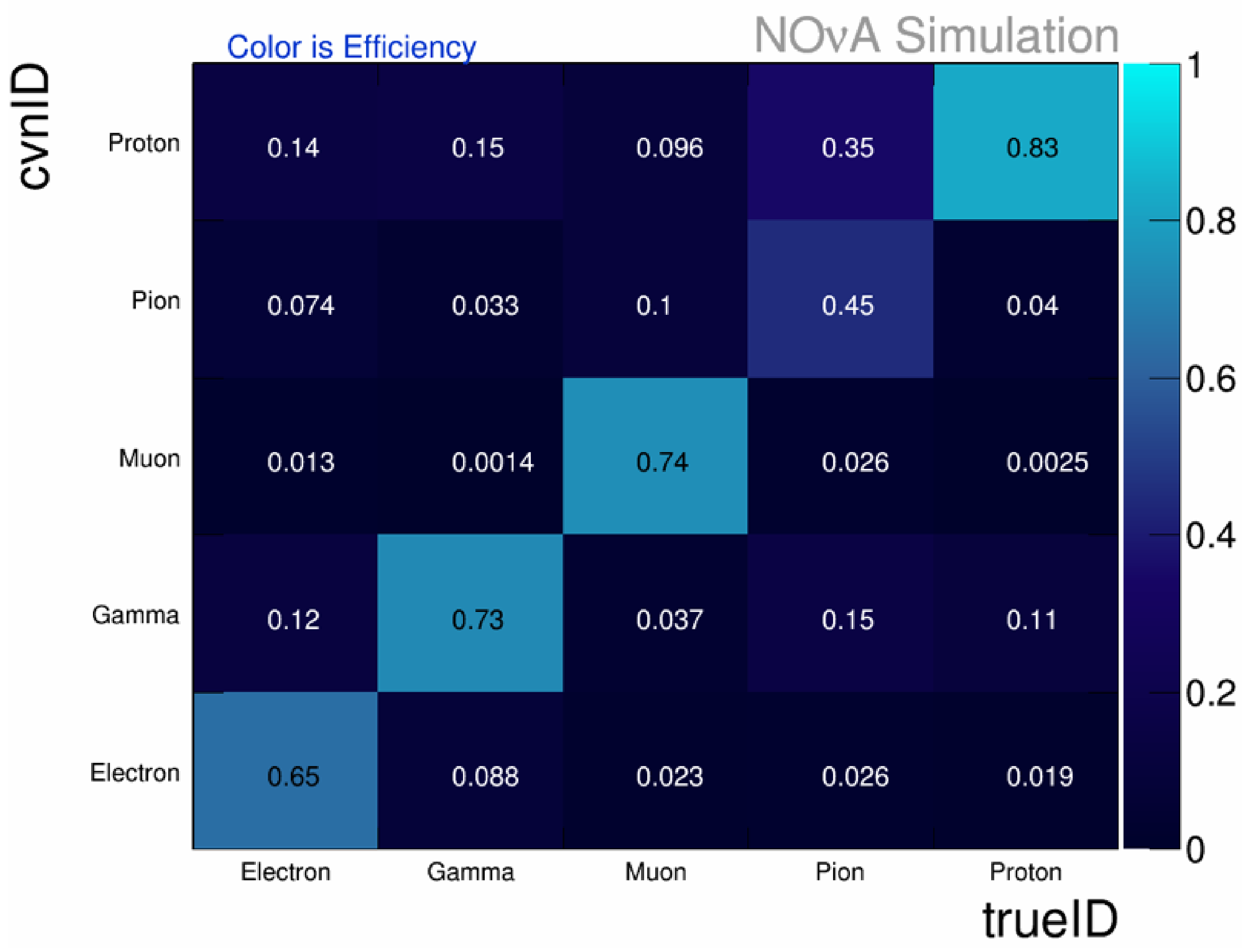




\section{Utilizing Context}

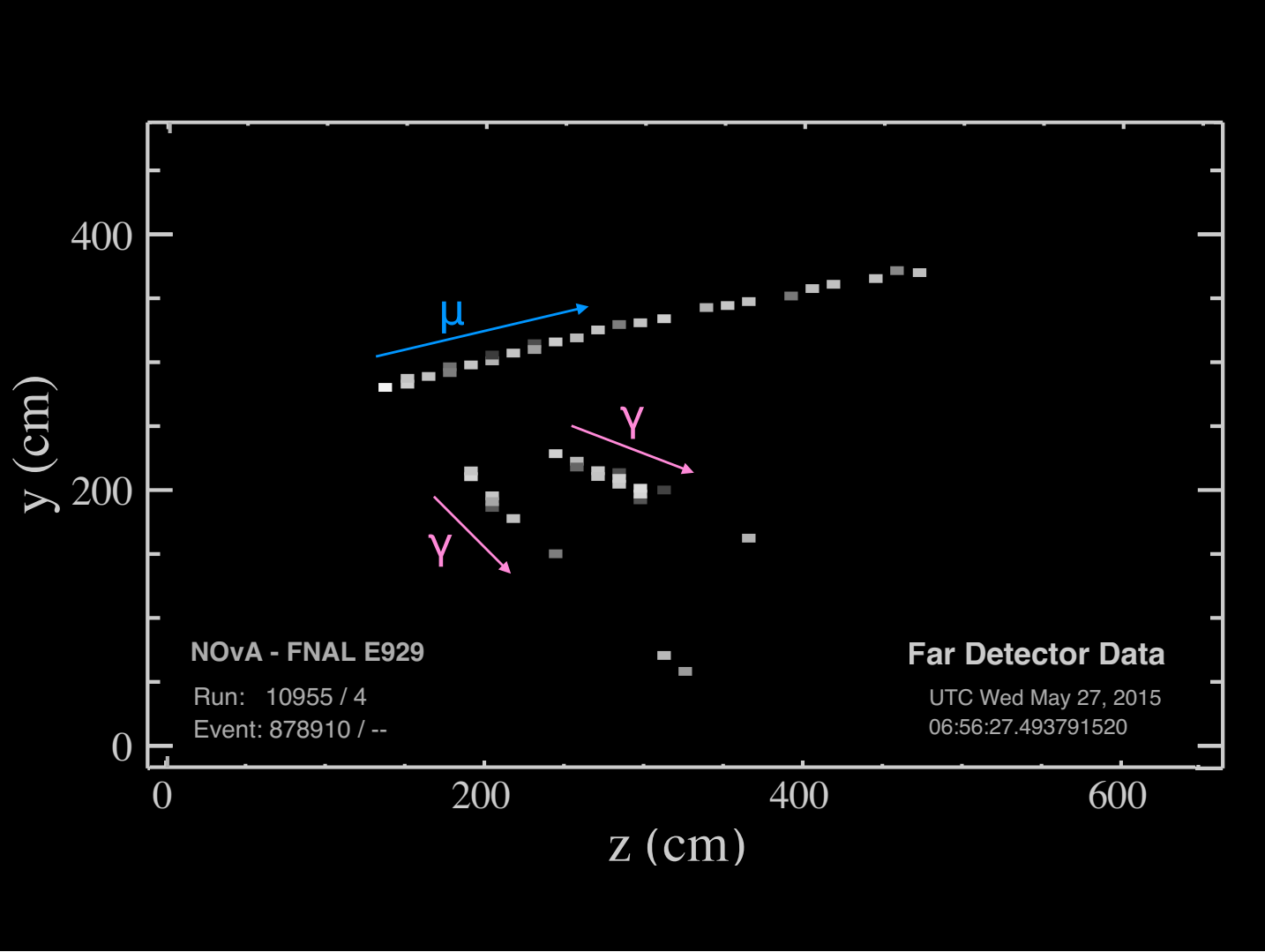

Showing the network the entire event teaches the network contextual information.

Particularly useful in the classification of photons.

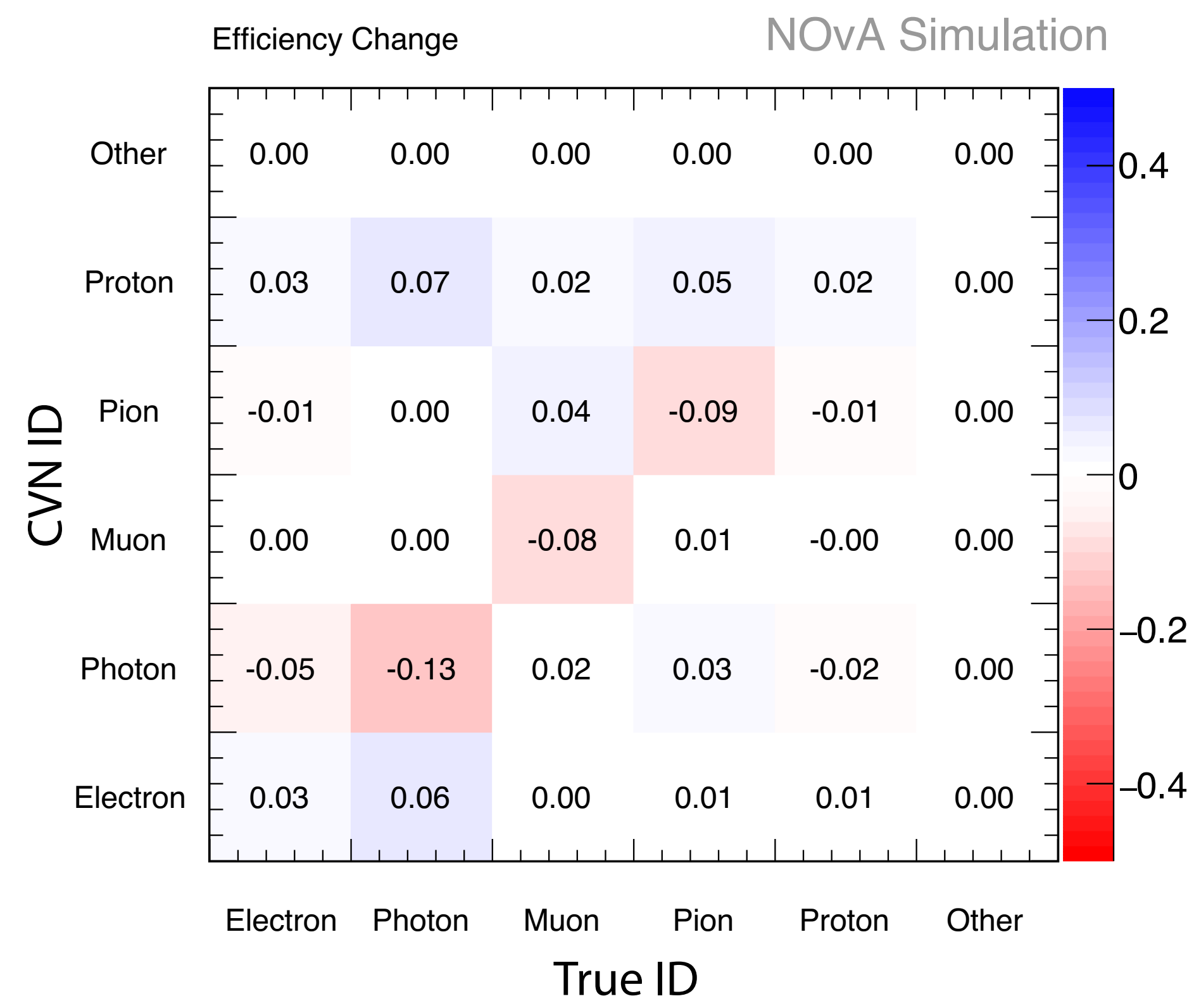

The change in efficiency for each category from removing context information. 


\section{Example Data Check: $\pi^{0}$ Mass Peak}
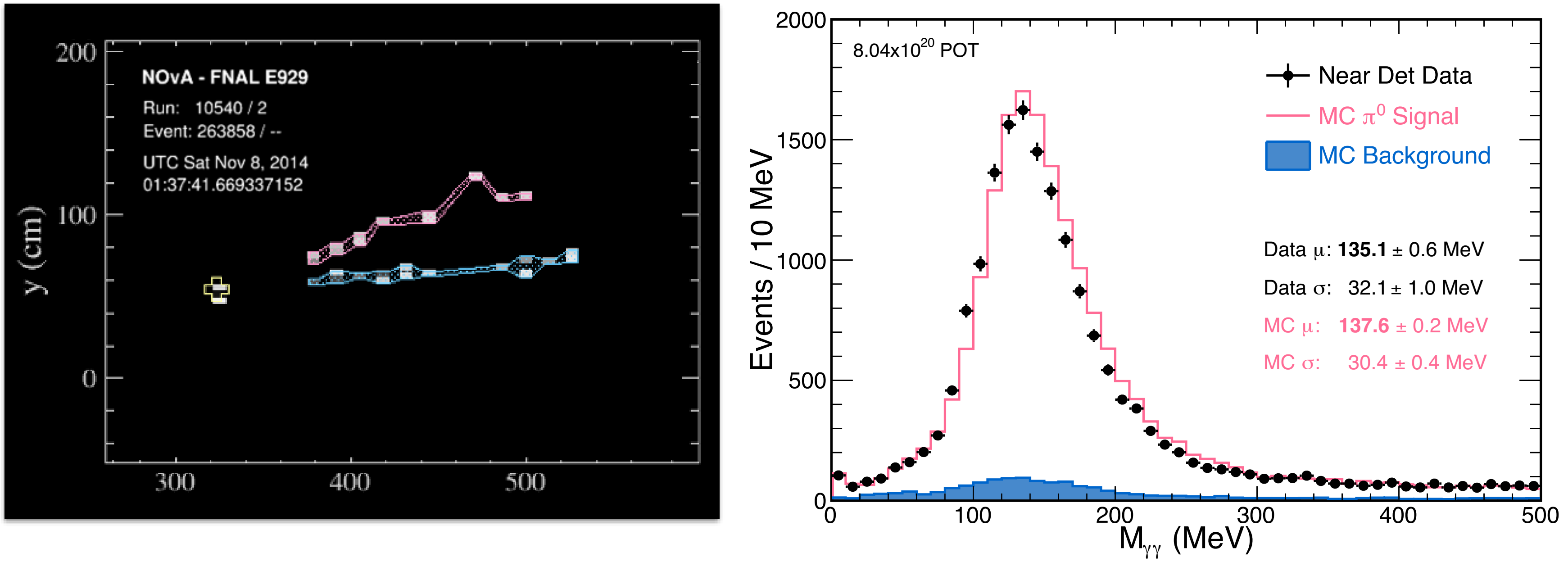

$\Pi^{0}$ mass reconstructed using invariant mass of pairs of photons identified using the single particle classifier. 


\section{Reconstruction Caveats}

Single particle classifier is dependent on the quality of the already existing clusters.
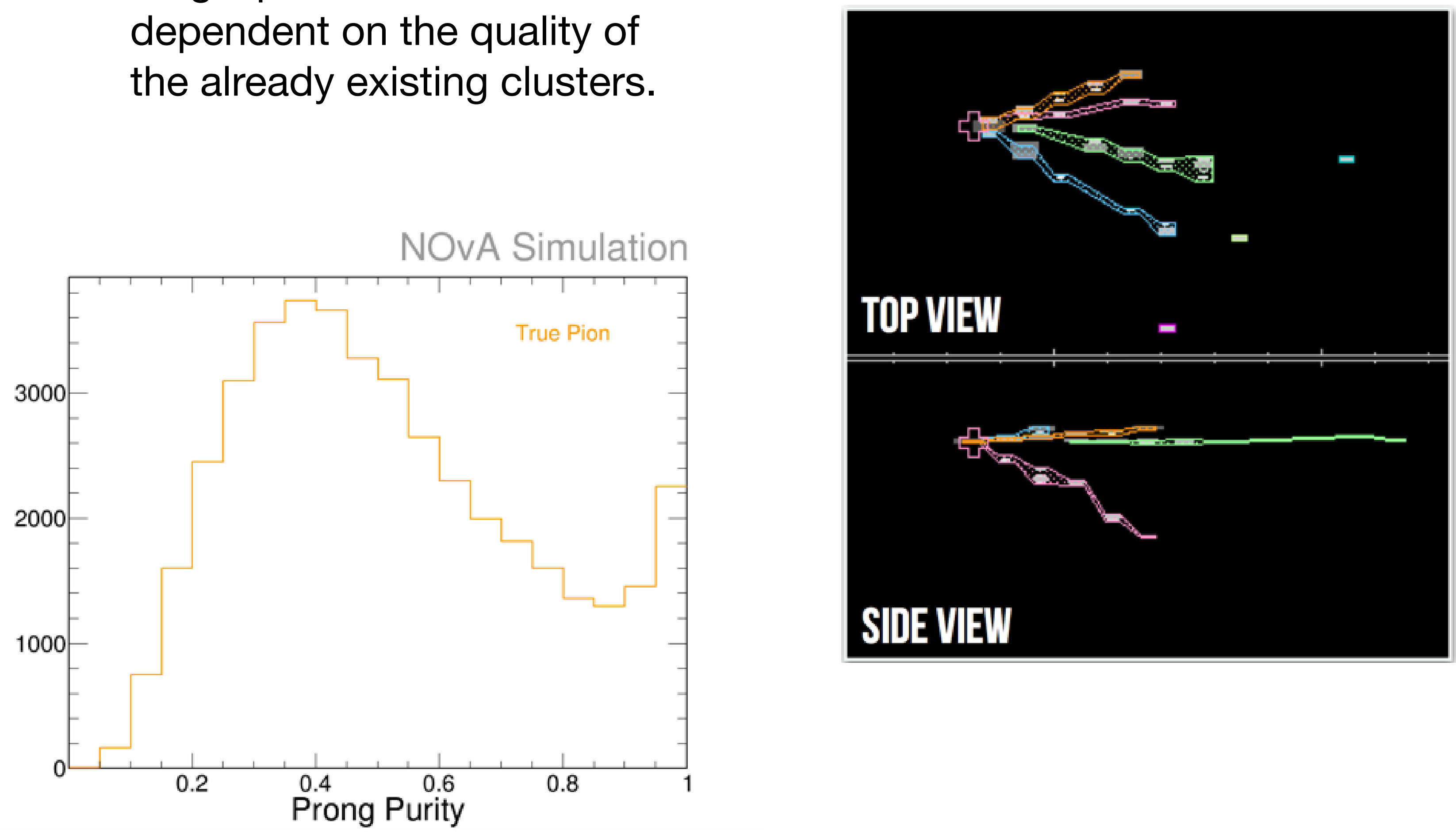


\section{Full Event Reconstruction}

Cluster and classify particles simultaneously using instance aware semantic segmentation.

A network reconstructs an event hit by hit.

Bounding Boxes - builds bounding boxes aiming to contain a single particle.

Labels - A softmax function is used to classify the particle in each box.

Clustering - Pixel by pixel clusters are defined to closely contain single particles.

Exploring different inputs to the network to improve clustering
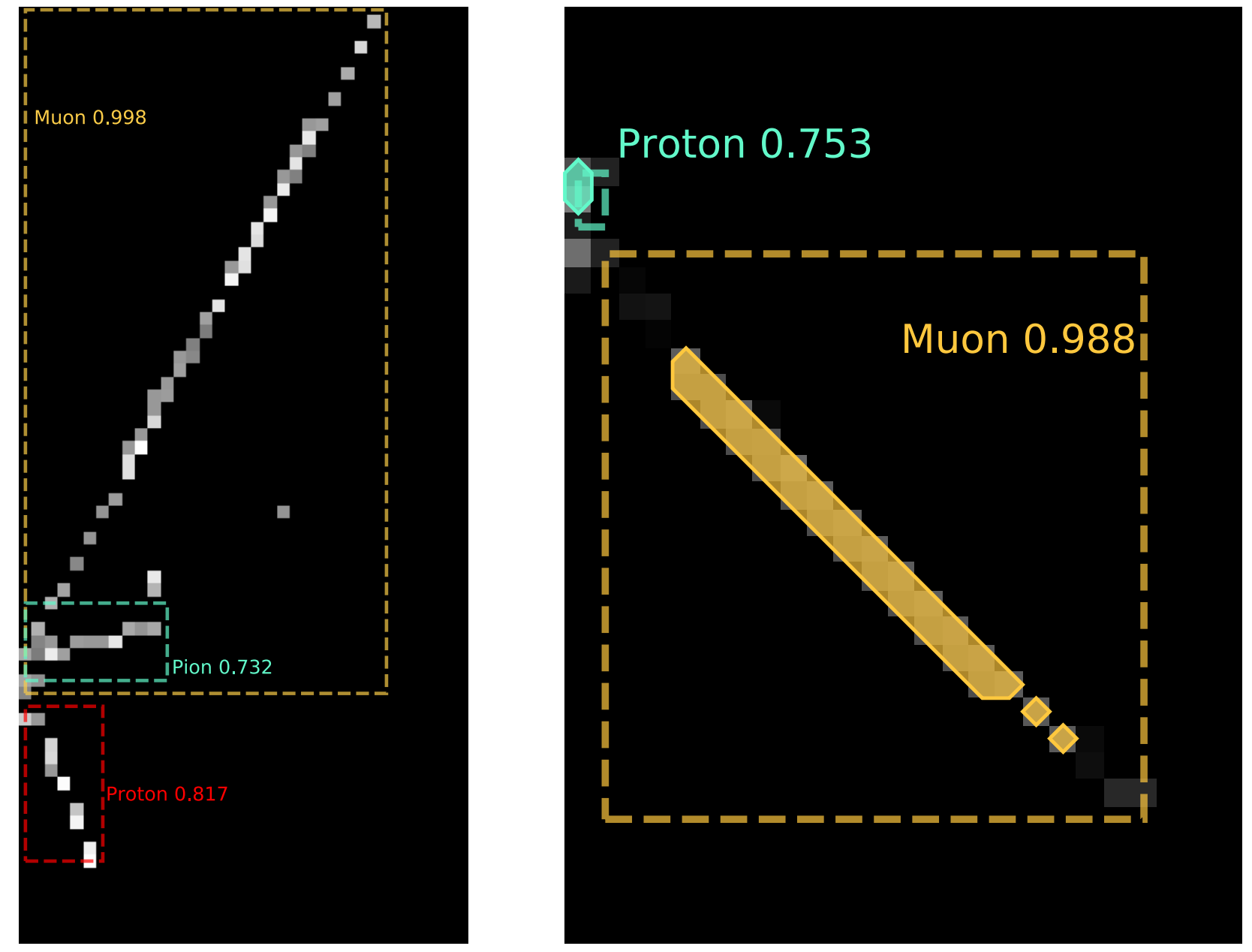
performance. 


\section{Conclusion}

Training on neutrinos and anti-neutrinos separately yields the largest improved for event classification.

Giving contextual information improves performance of single particle classification.

Data-driven methods are used to check the performance of the networks.

Developing a network for full event reconstruction.

$$
\begin{aligned}
& \text { http://nusoft.fnal.gov/nova/public/nova-events/ } \\
& \text { Can you beat our } \\
& \text { neural nets? }
\end{aligned}
$$




\section{Backup}




\section{Signature Data Events}

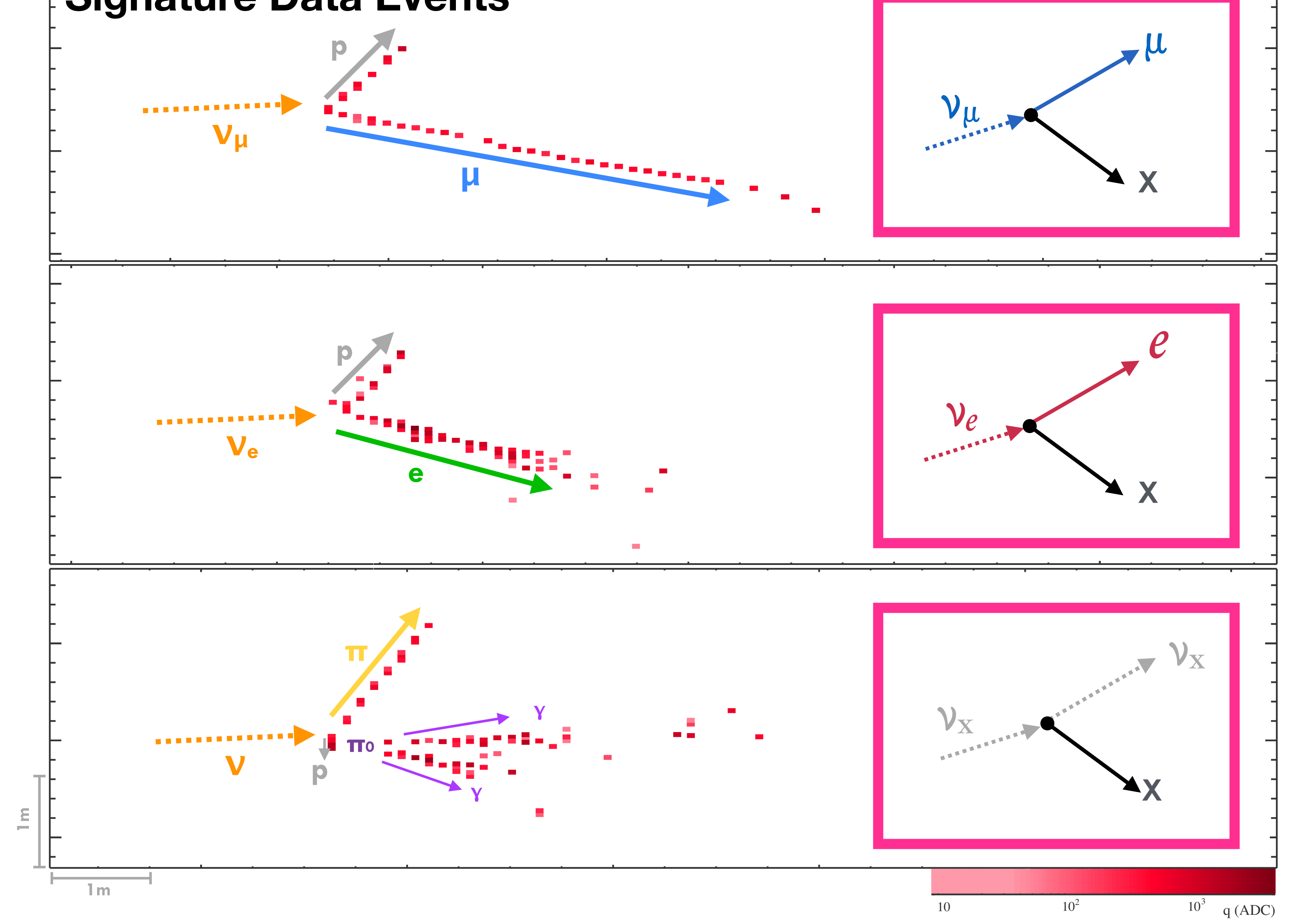






\section{NOvA - FNAL E929}

Run: 14828 / 38

Event: 192569 / --

UTC Tue Apr 22, 2014 21:41:51.422846016

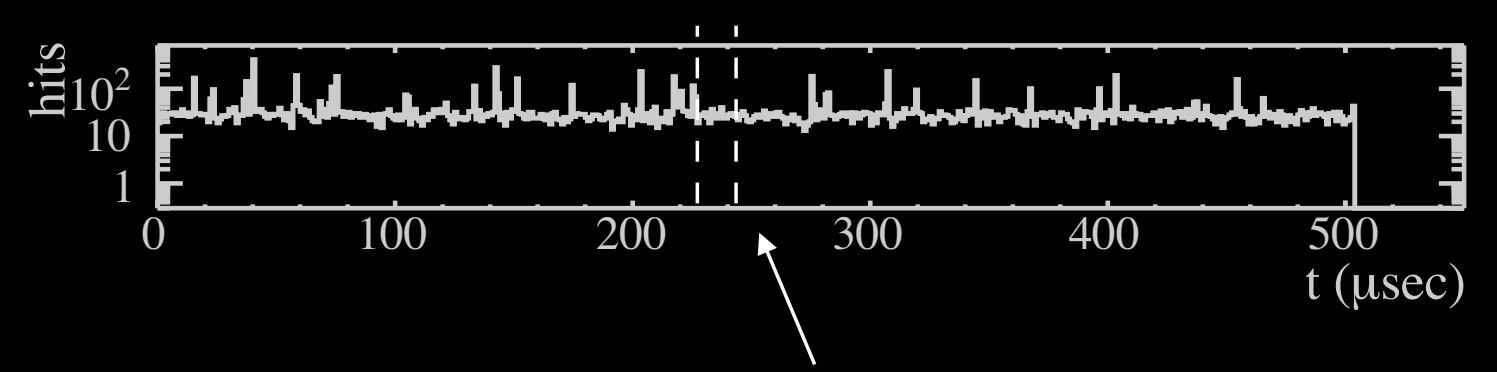

\section{beam window}

Micah Groh

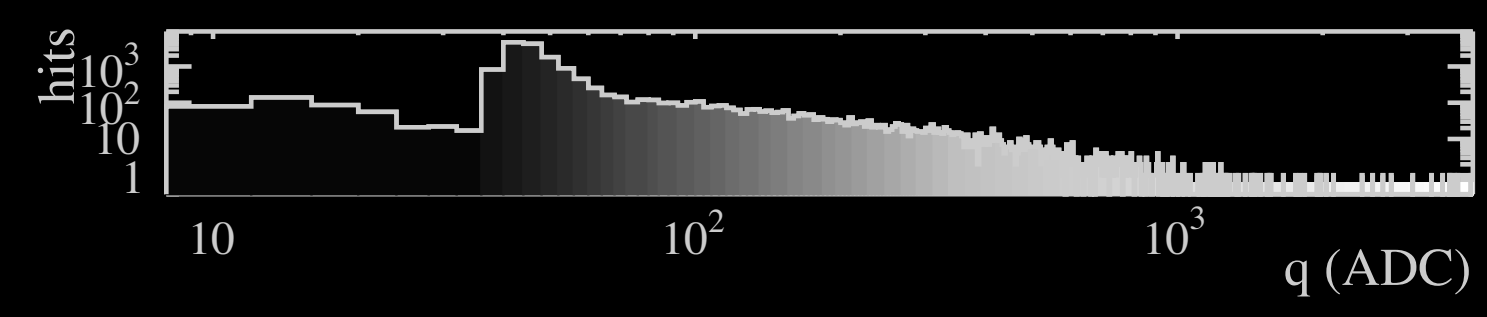

New Perspectives 2018 


\section{NOvA Events}

Separate hits by time and space

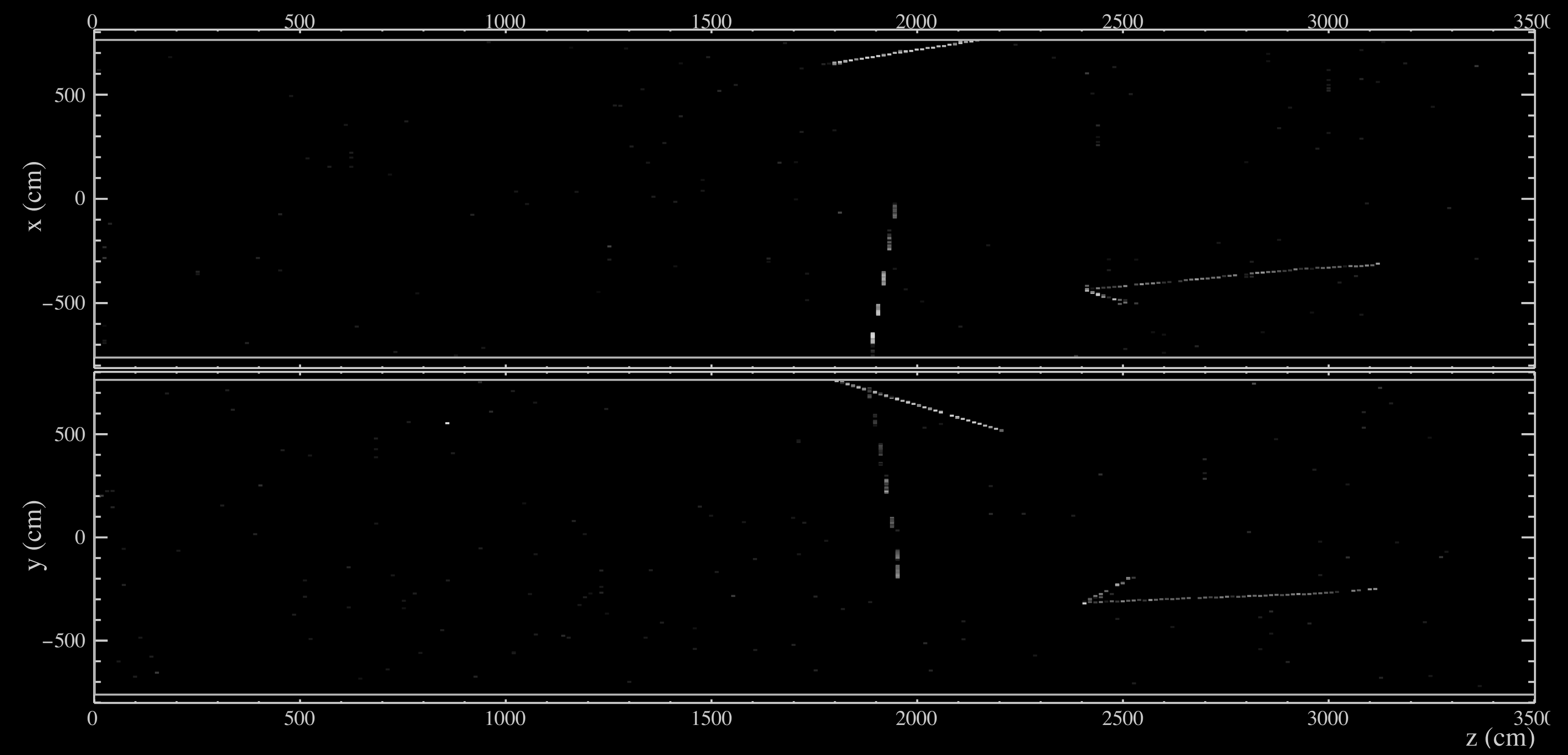

NOvA - FNAL E929

Run: 14828 / 38

Event: 192569 / --

UTC Tue Apr 22, 2014

21:41:51.422846016
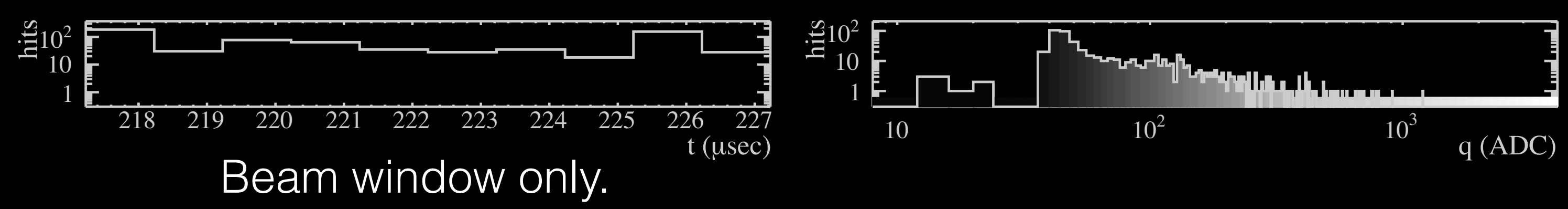


\section{Neural Networks}

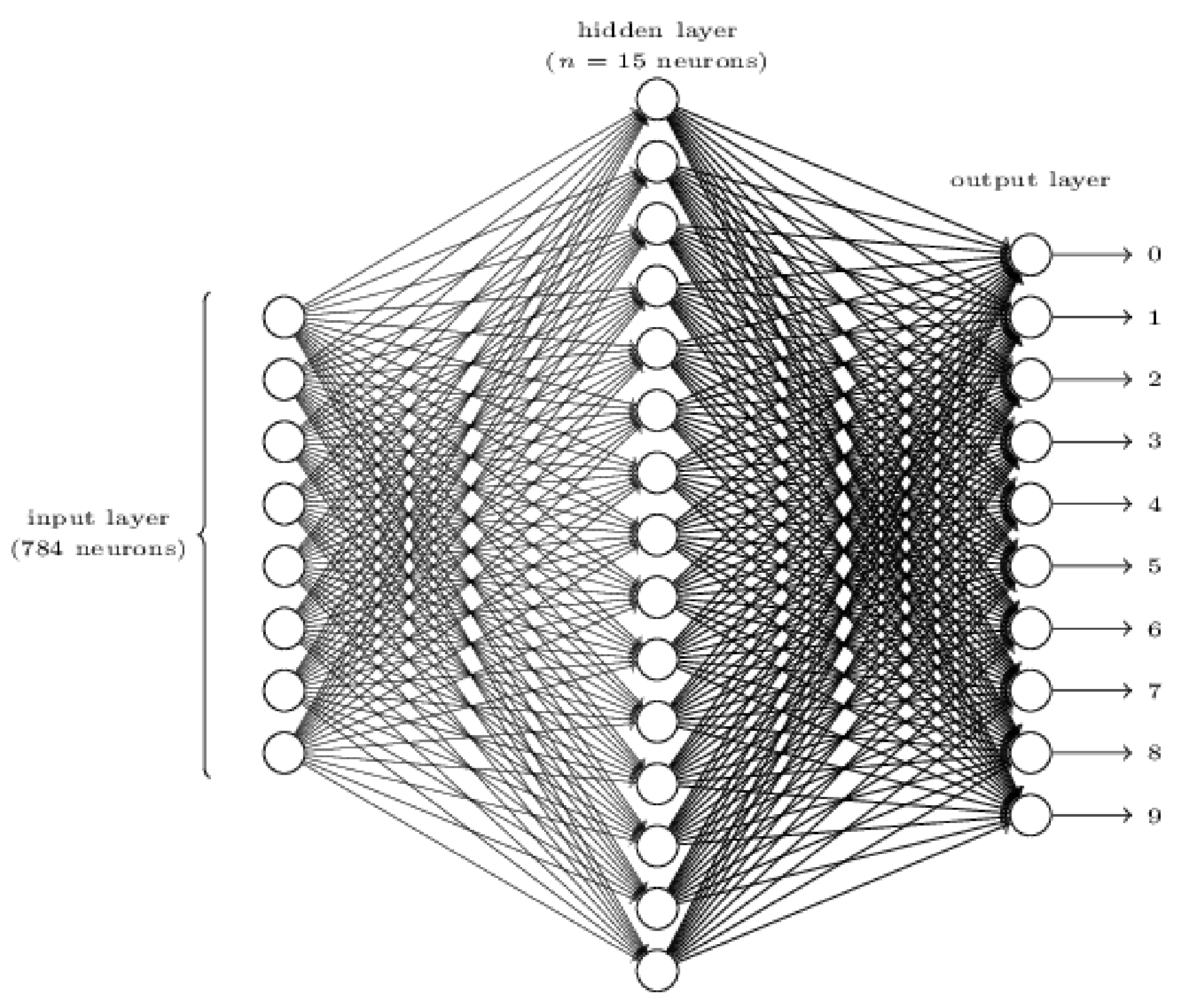

Identify neutrino flavor using neural networks.

Artificial Neural Network (ANN) consists of multiple layers of neurons.

Each neuron represents a function using the values from the previous layer.

Output layer has scores for each category. 


\section{Convolutions and Pooling}
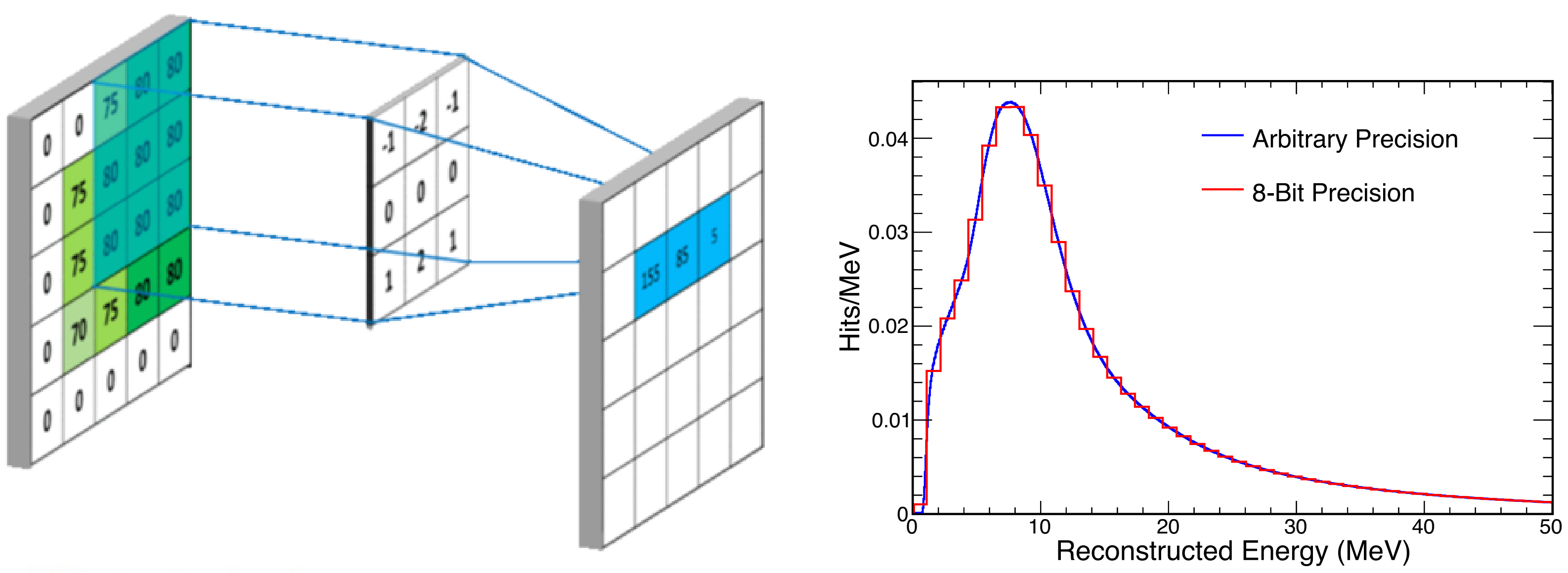

Single depth slice

\begin{tabular}{|c|c|c|c|}
\hline 1 & 1 & 2 & 4 \\
\hline 5 & 6 & 7 & 8 \\
\hline 3 & 2 & 1 & 0 \\
\hline 1 & 2 & 3 & 4 \\
\hline
\end{tabular}

max pool with $2 \times 2$ filters and stride 2

\begin{tabular}{|l|l|}
\hline 6 & 8 \\
\hline 3 & 4 \\
\hline
\end{tabular}




\section{Pooling}

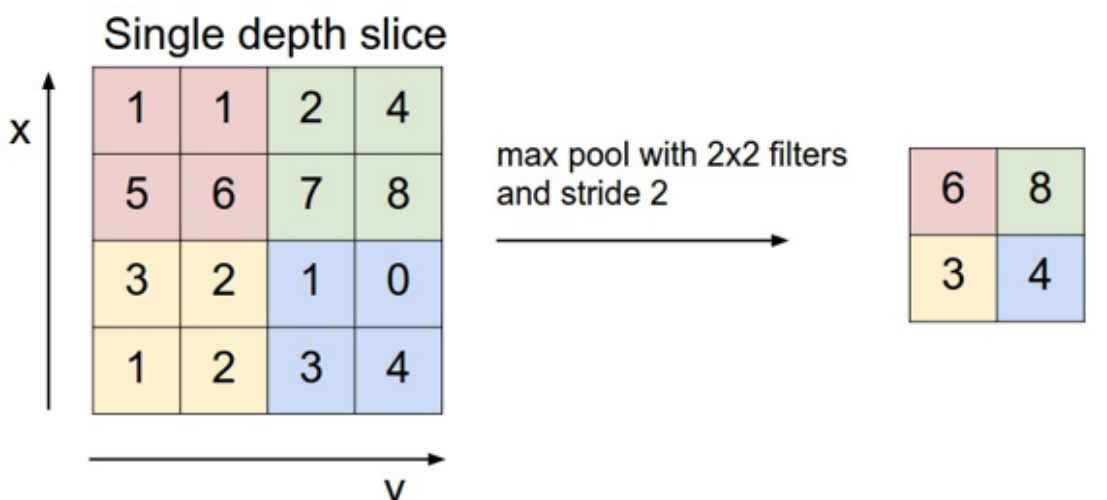

\section{Dropout}

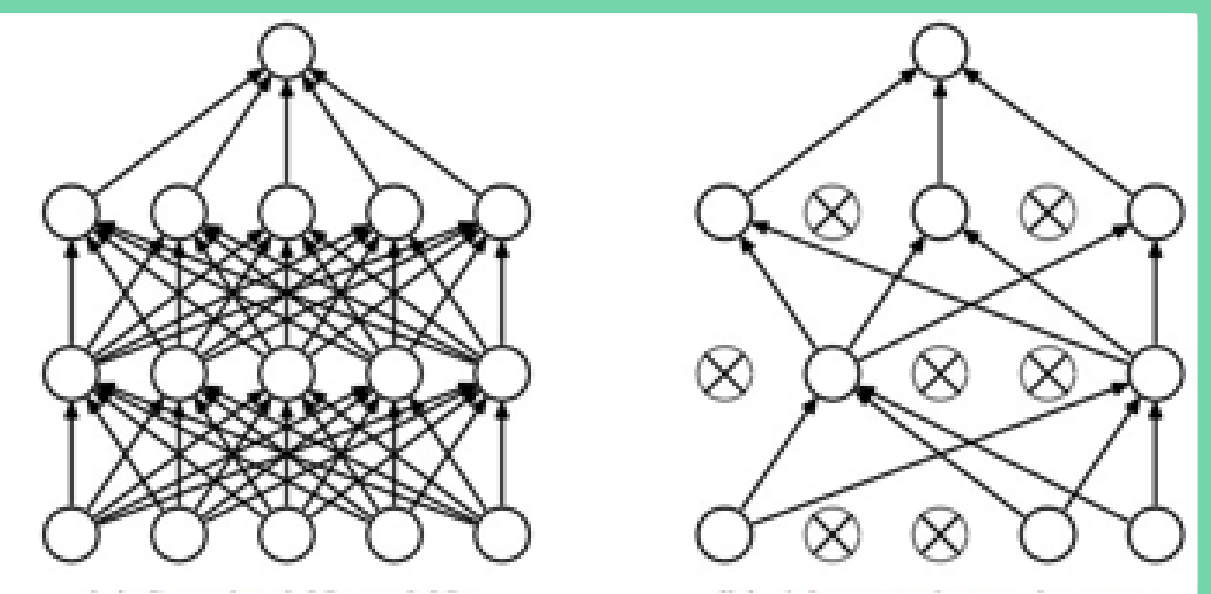

\section{Inception module}

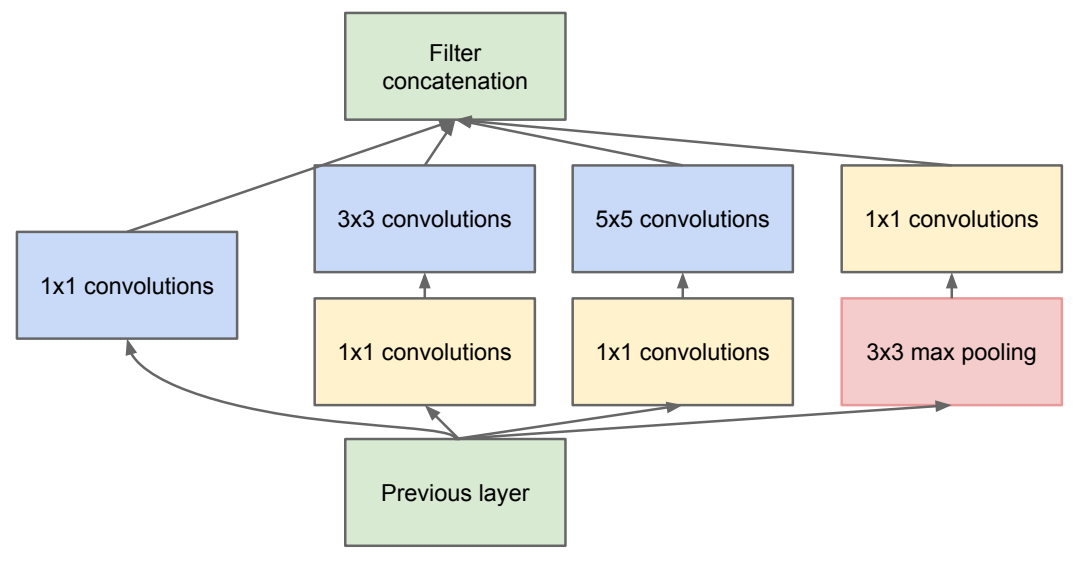

\section{Convolution}

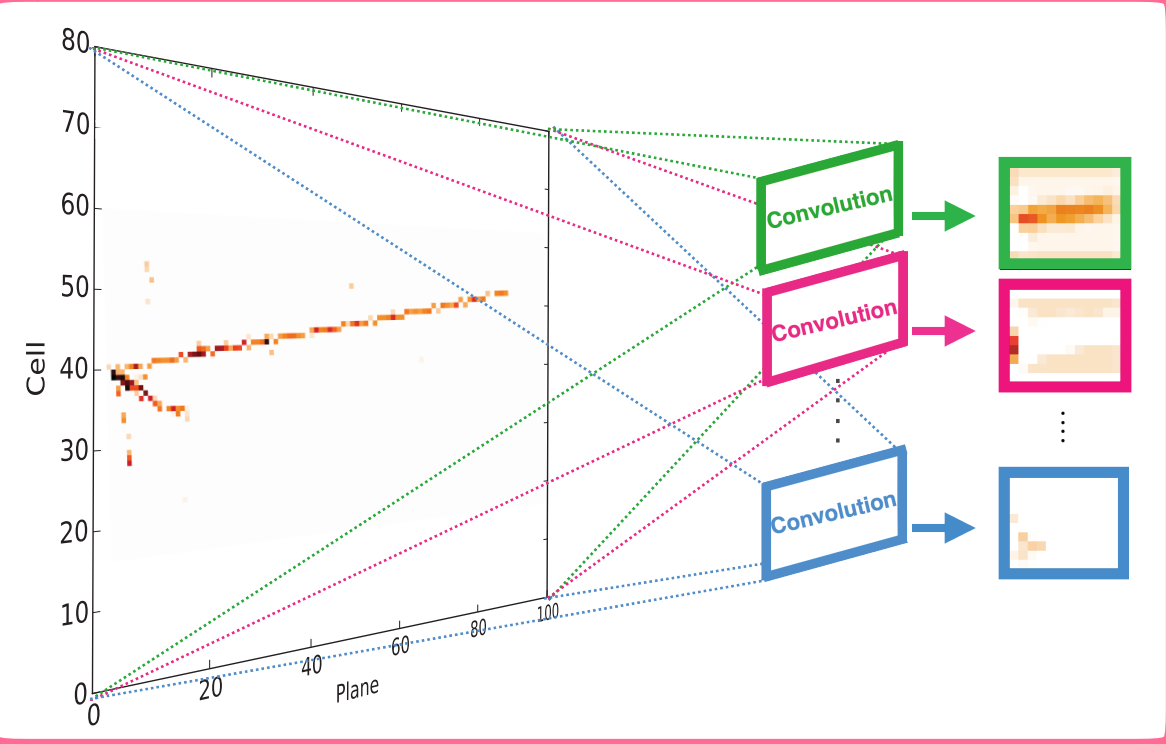

\section{Inception output}

\begin{tabular}{llll|l|l|l|l|l|l}
\hline & & & & & & & & \\
\hline & & & & & & & & \\
\hline & & & & & & & & \\
\hline & & & & & & & & \\
\hline & & & & & & & & \\
\hline & & & & & $=$ & & & \\
\hline & & & & & & & & \\
\hline & & & & & & - & & \\
\hline
\end{tabular}




\section{Training}

\section{x4.7 million}

\section{HELLO \\ Ve CC}

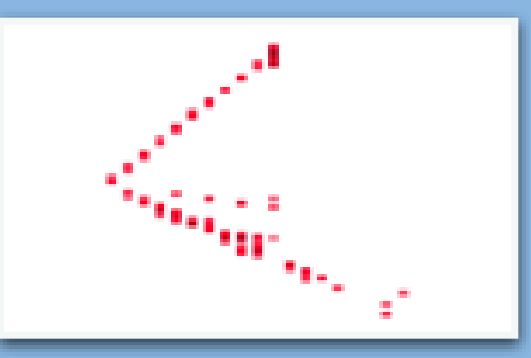

:
Training Sample

Test Sample

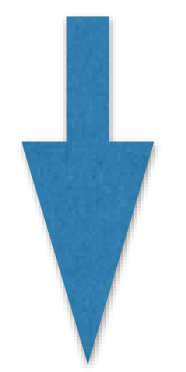

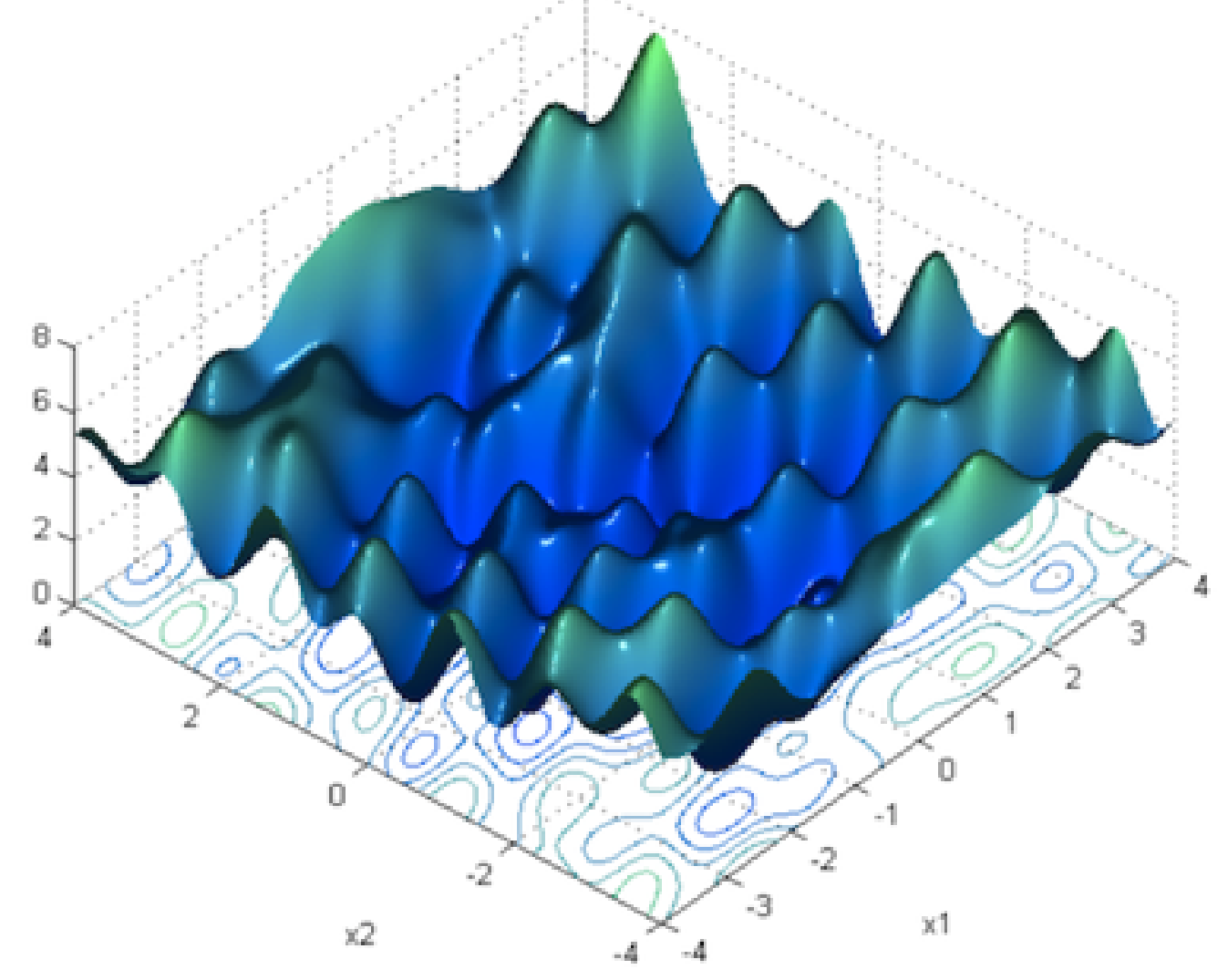






\section{Traditional Reconstruction}

Group all hits with a common origin, the same neutrino interaction or cosmic.

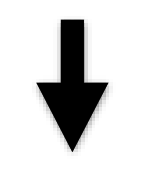

Reconstruct the global interaction vertex.

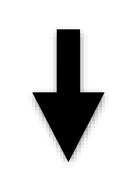

Cluster hits belonging to the same particle.

\section{$\downarrow$}

Match clusters across views to make 3D prongs
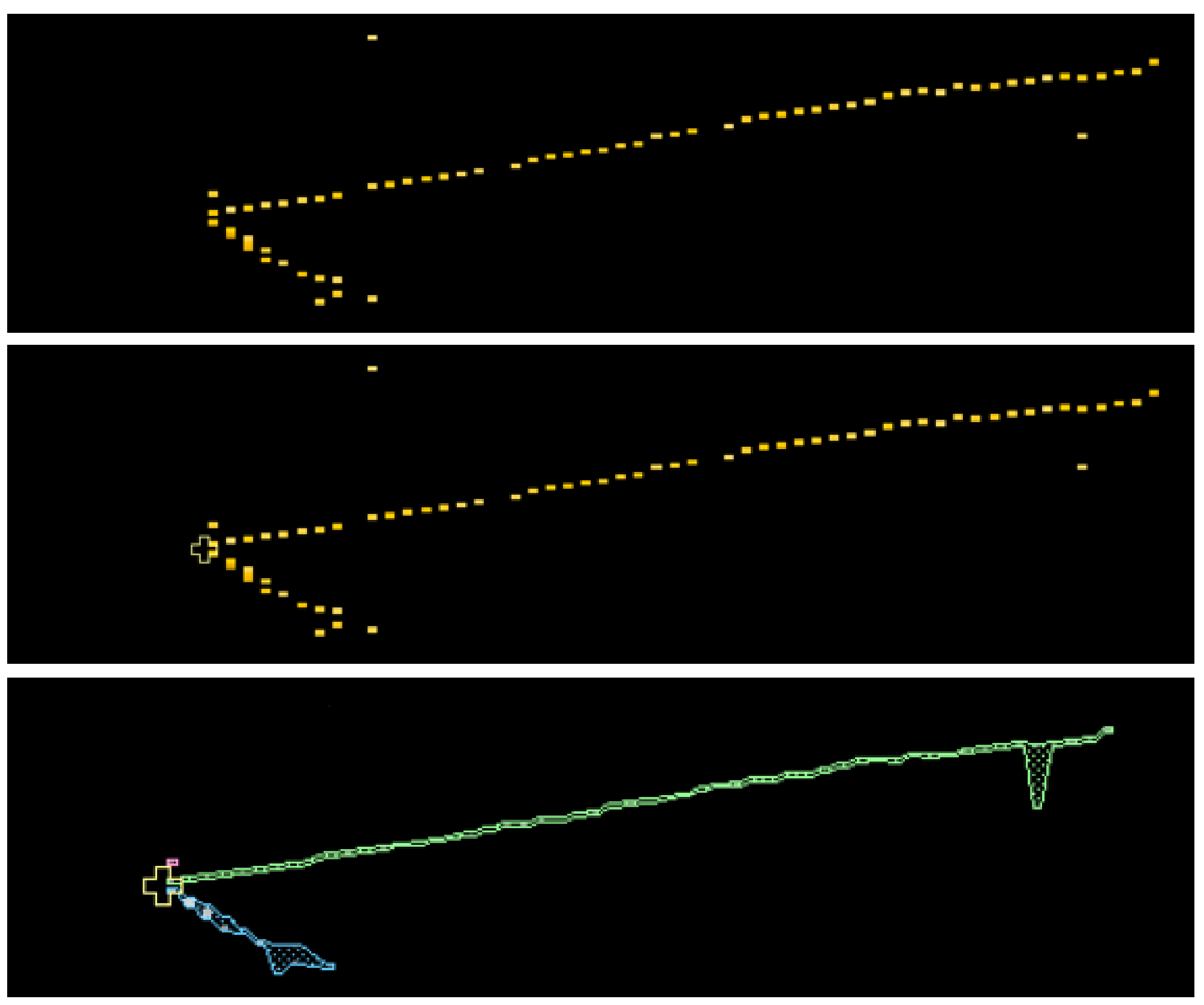


\section{Event Identification}
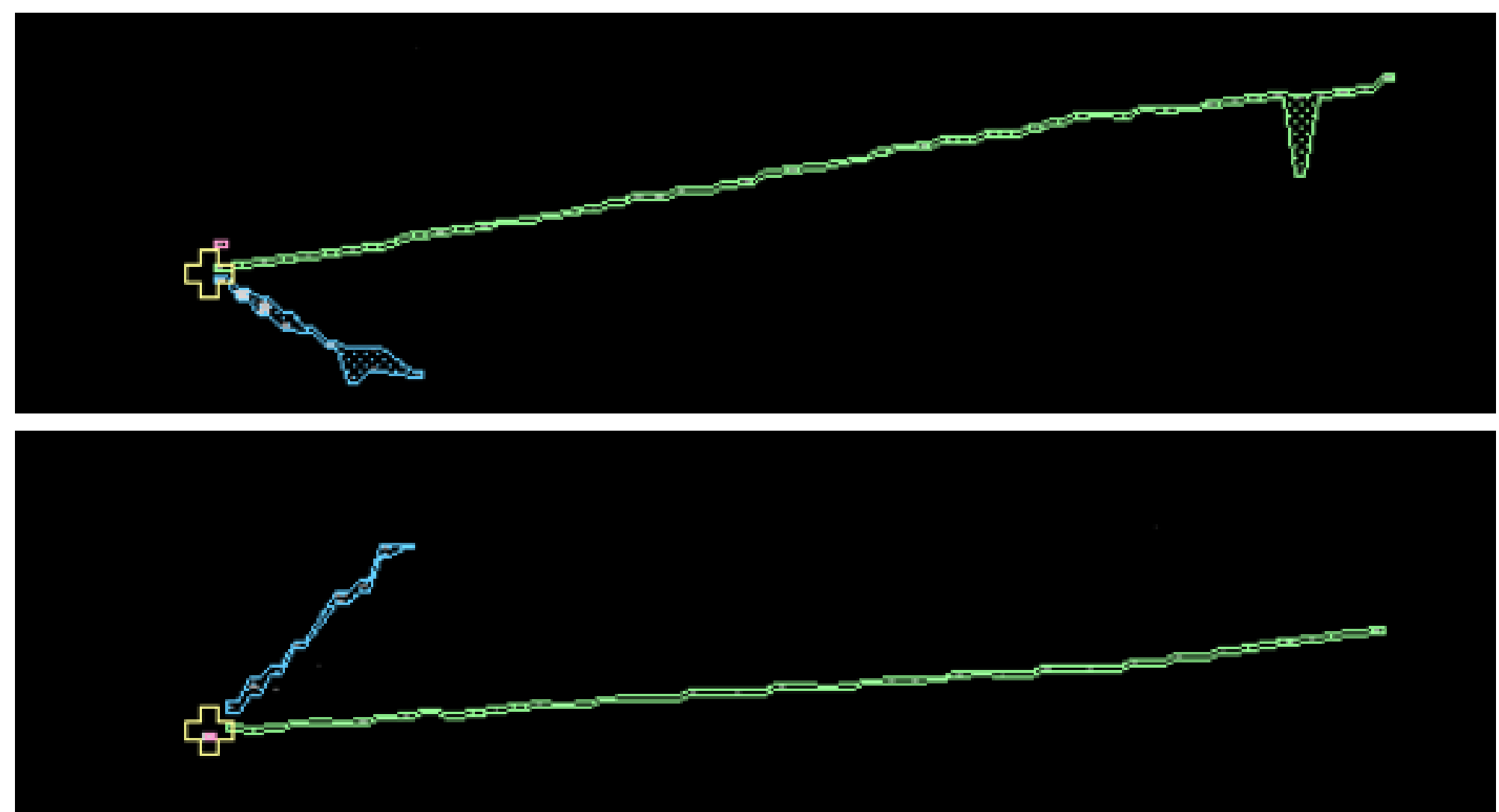

Extracted features from geometrical reconstruction used in a neural net.

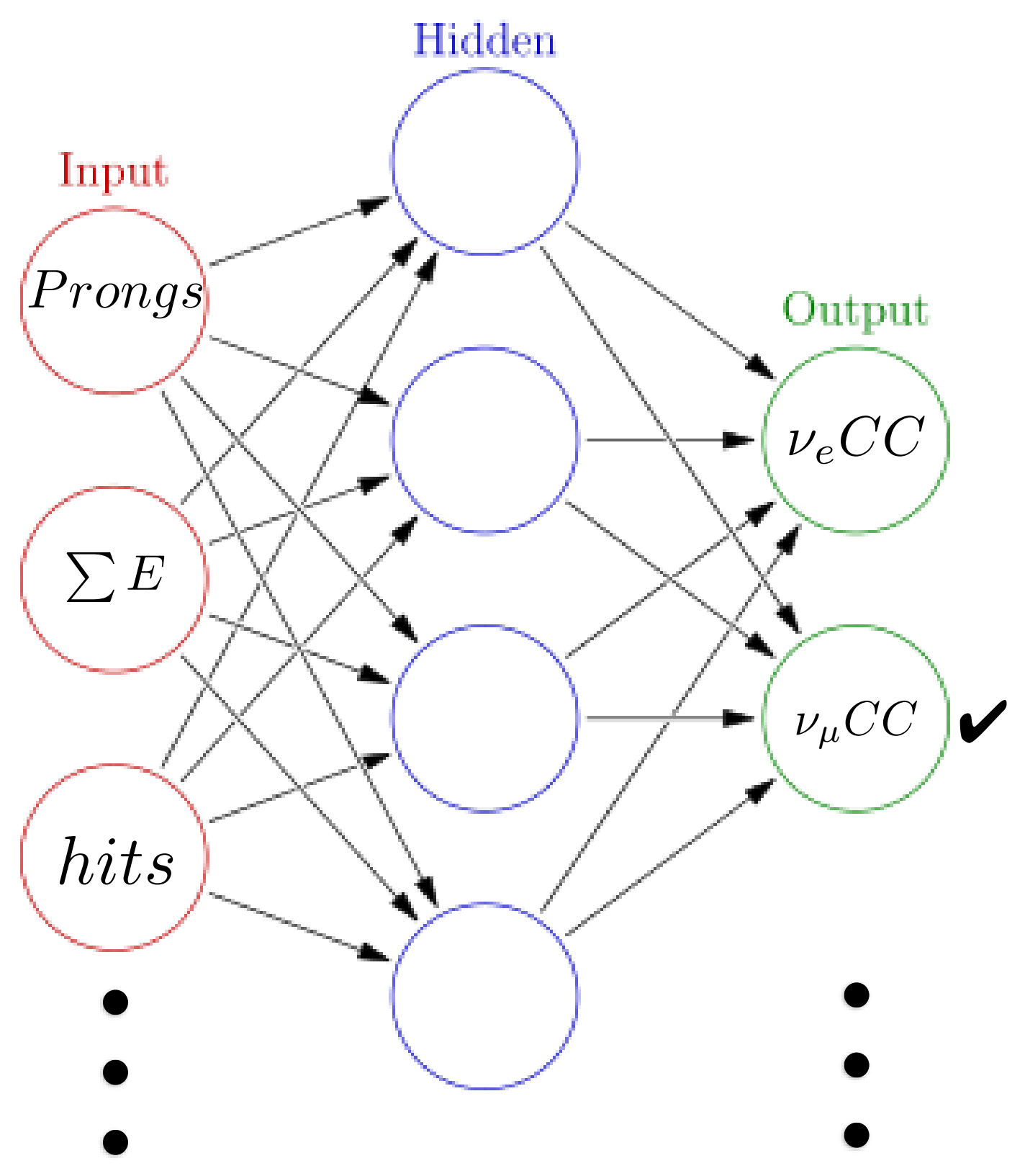

Output is the interaction type. 


\section{NOvA Features}
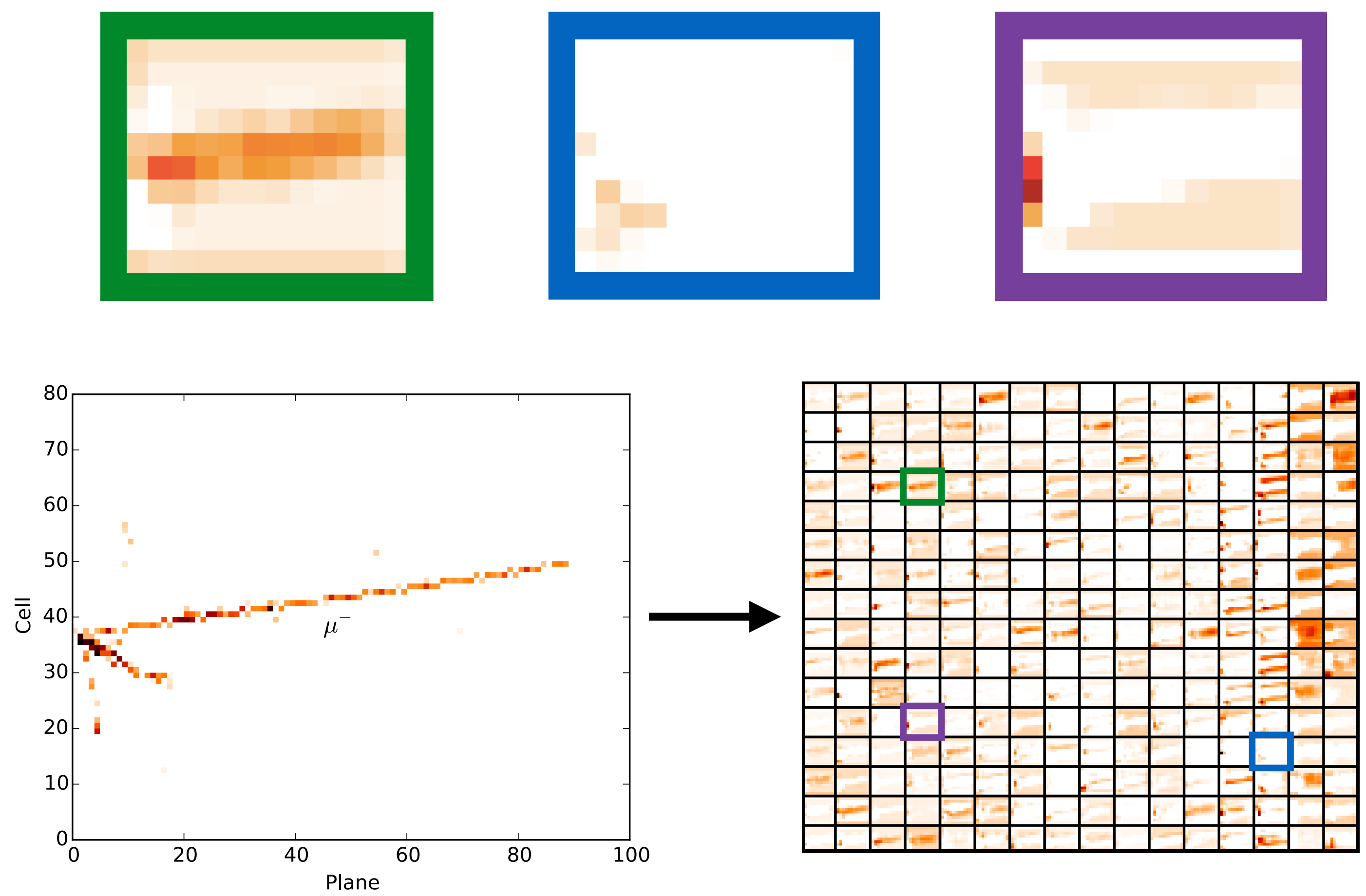


\section{NOvA Features}
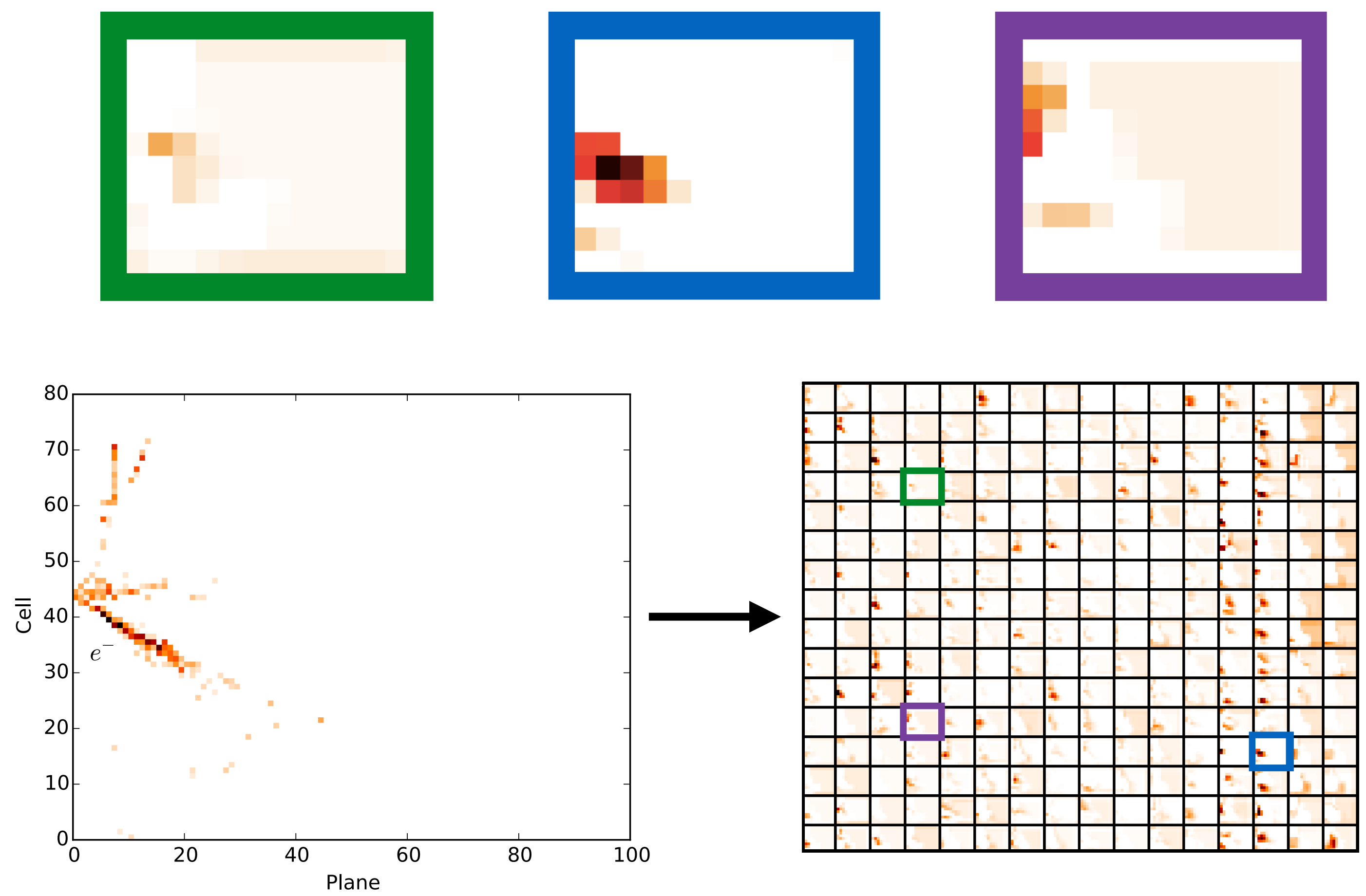


\section{Prong CVN}

Broad categories looking at just electromagnetic and hadronic particles as well as muons.
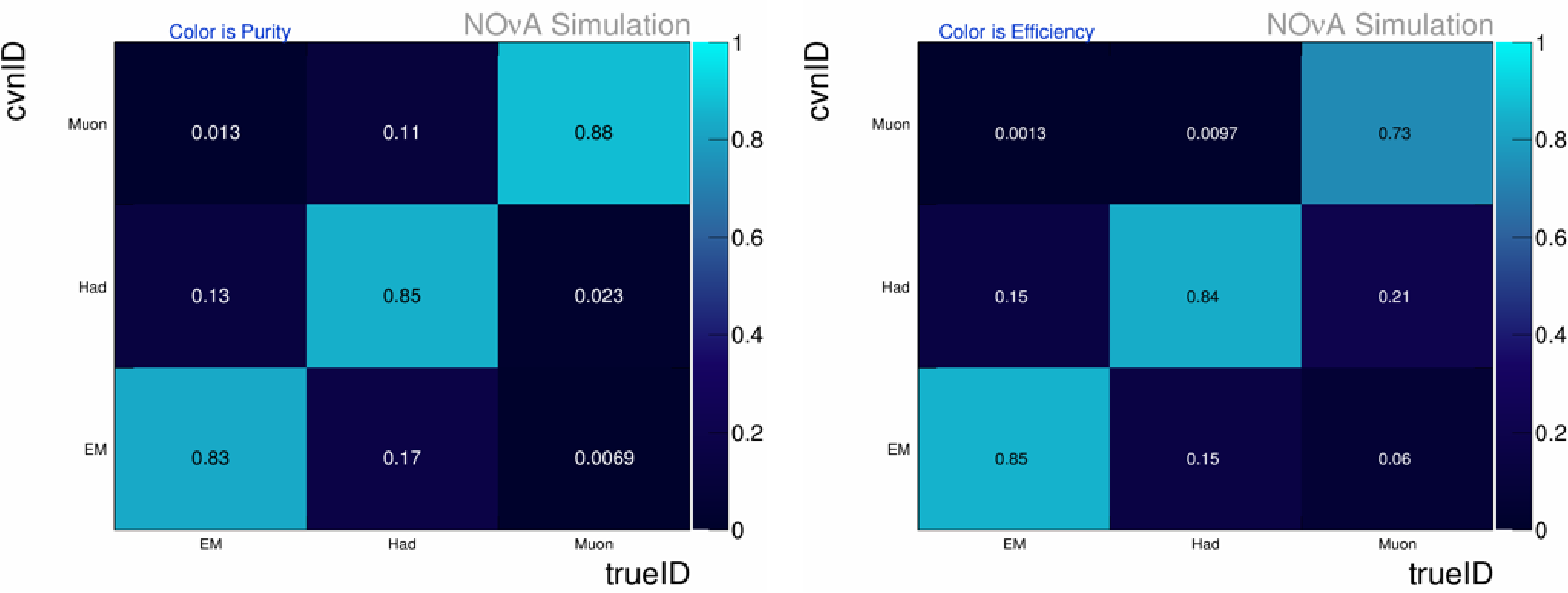


\section{Nue Energy Estimator}
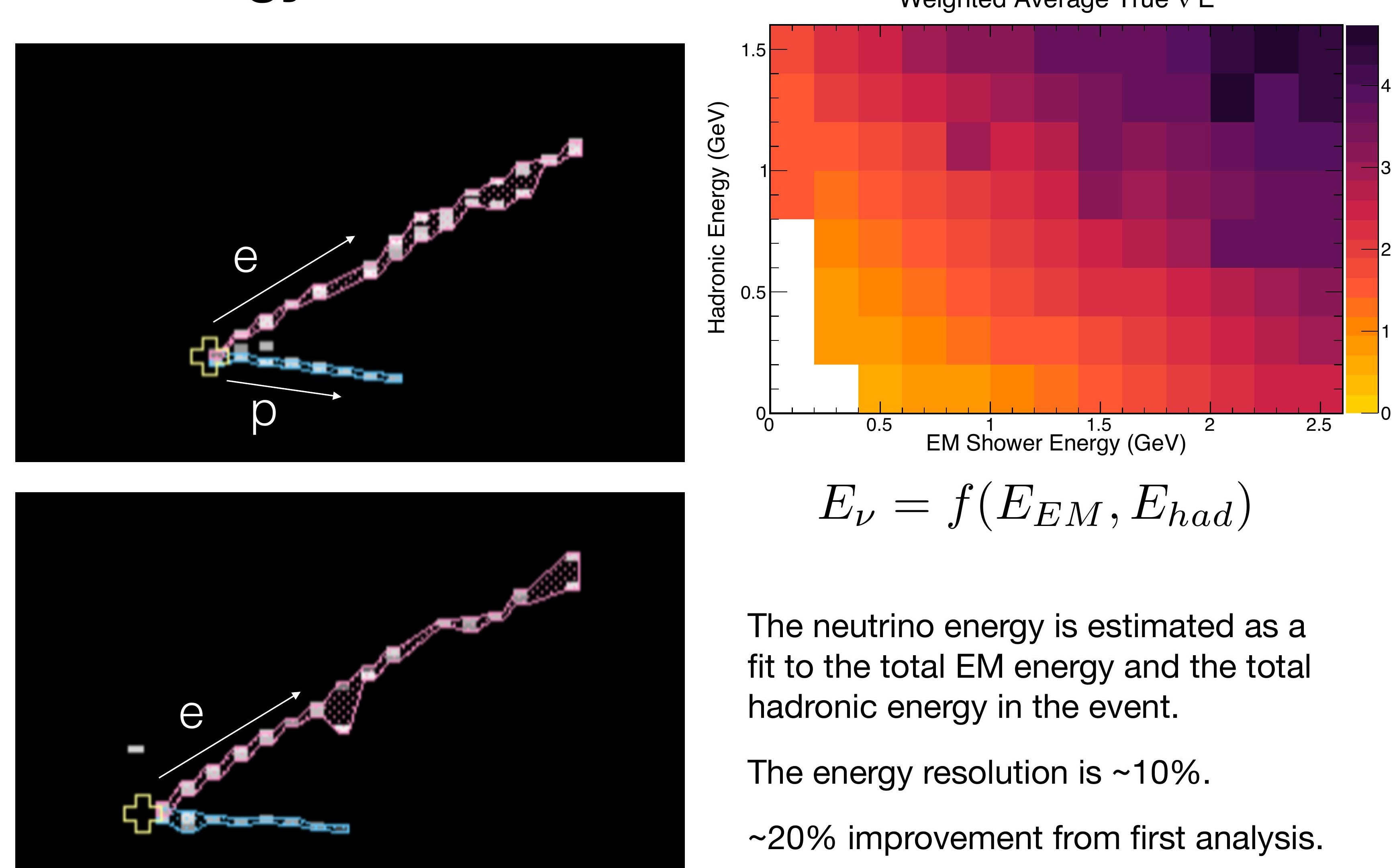

ש

The neutrino energy is estimated as a fit to the total EM energy and the total hadronic energy in the event.

The energy resolution is $\sim 10 \%$.

$\sim 20 \%$ improvement from first analysis. 


\section{Full Event Reconstruction}
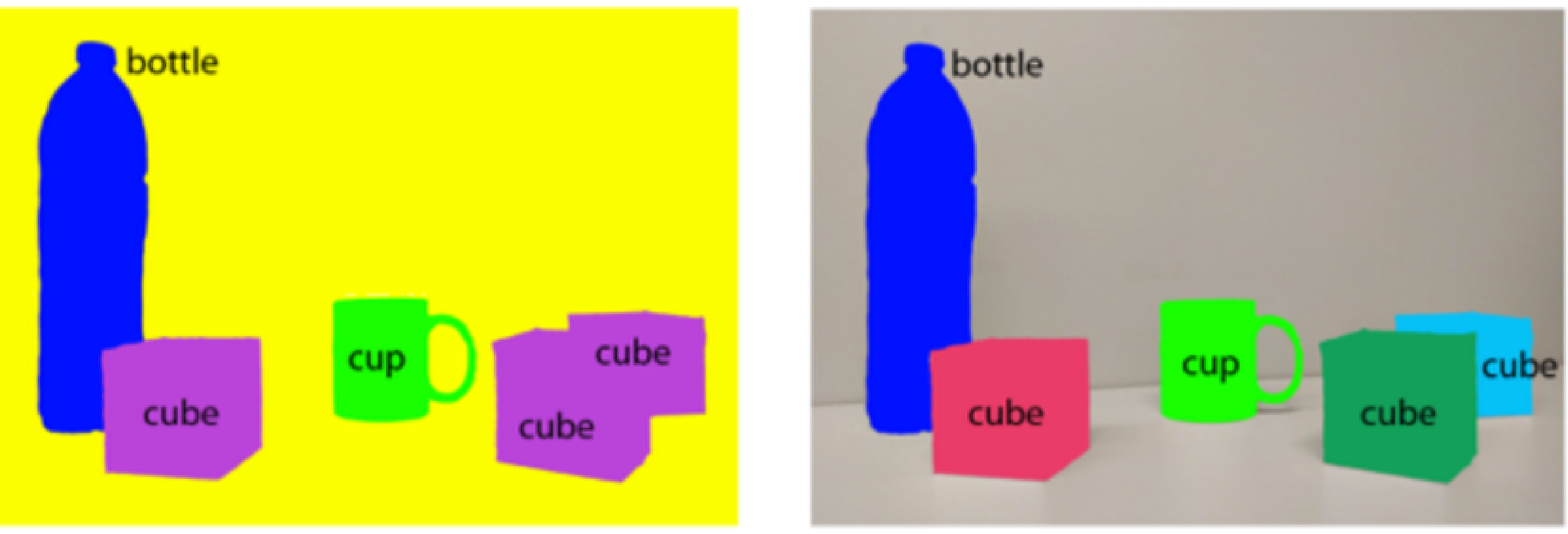

Semantic Segmentation

Train a network for pixel by pixel classification

Instance Segmentation

Network clusters hits and assigns a label to each cluster 